\title{
Unified gas-kinetic wave-particle methods IV: multi-species gas mixture and plasma transport
}

\author{
Chang Liu ${ }^{1,2}$ and Kun $\mathrm{Xu}^{1,3^{*}}$ (1)
}

\author{
*Correspondence: makxu@ust.hk \\ 1 Department of Mathematics, Hong \\ Kong University of Science and \\ Technology, Hong Kong, China \\ ${ }^{3}$ Shenzhen Research Institute, Hong \\ Kong University of Science and \\ Technology, Shenzhen 518057, \\ China \\ Full list of author information is \\ available at the end of the article
}

\begin{abstract}
In this paper, we extend the unified gas-kinetic wave-particle (UGKWP) methods to the multi-species gas mixture and multiscale plasma transport. The construction of the scheme is based on the direct modeling on the mesh size and time step scales, and the local cell's Knudsen number determines the flow physics. The proposed scheme has the multiscale and asymptotic complexity diminishing properties. The multiscale property means that according to the cell's Knudsen number the scheme can capture the non-equilibrium flow physics when the cell size is on the kinetic mean free path scale, and preserve the asymptotic Euler, Navier-Stokes, and magnetohydrodynamics $(\mathrm{MHD})$ when the cell size is on the hydrodynamic scale and is much larger than the particle mean free path. The asymptotic complexity diminishing property means that the total degrees of freedom of the scheme reduce automatically with the decreasing of the cell's Knudsen number. In the continuum regime, the scheme automatically degenerates from a kinetic solver to a hydrodynamic solver. In the UGKWP, the evolution of microscopic velocity distribution is coupled with the evolution of macroscopic variables, and the particle evolution as well as the macroscopic fluxes is modeled from a time accumulating solution of kinetic scale particle transport and collision up to a time step scale. For plasma transport, the current scheme provides a smooth transition from particle-in-cell (PIC) method in the rarefied regime to the magnetohydrodynamic solver in the continuum regime. In the continuum limit, the cell size and time step of the UGKWP method are not restricted by the particle mean free path and mean collision time. In the highly magnetized regime, the cell size and time step are not restricted by the Debye length and plasma cyclotron period. The multiscale and asymptotic complexity diminishing properties of the scheme are verified by numerical tests in multiple flow regimes.
\end{abstract}

Keywords: Unified gas-kinetic wave-particle method, Multiscale modeling, Gas mixture, Plasma transport (c) The Author(s). 2021 Open Access This article is licensed under a Creative Commons Attribution 4.0 International License, which permits use, sharing, adaptation, distribution and reproduction in any medium or format, as long as you give appropriate credit to the original author(s) and the source, provide a link to the Creative Commons licence, and indicate if changes were made. The images or other third party material in this article are included in the article's Creative Commons licence, unless indicated otherwise in a credit line to the material. If material is not included in the article's Creative Commons licence and your intended use is not permitted by statutory regulation or exceeds the permitted use, you will need to obtain permission directly from the copyright holder. To view a copy of this licence, visit http://creativecommons.org/licenses/by/4.0/. 


\section{Introduction}

Gas mixture and plasma widely exit in the universe and are extensively applied in the industry of aerospace, chemical, and nuclear engineering. Both gas mixture and plasma transport have multiscale nature. For the gas mixture, the flow regime varies from rarefied to continuum regimes according to the Knudsen number. In the rarefied regime, the fundamental governing equation is the multi-species Boltzmann equation [1], which resolves the flow physics on the particle mean free path and mean collision time scales. The complex five-fold integral collision operator of the Boltzmann equation makes both mathematical analysis and numerical simulation difficult. Therefore, many kinetic models have been proposed. For example, the McCormack model [2] linearizes the nonlinear collision term with the assumption for the distribution function to slightly deviate from equilibrium. The collision term of Andries-Aoki-Perthame (AAP) model [3] is approximated by a single relaxation term. Other modified models attempt to recover correct transport coefficients $[4,5]$. Although the kinetic equation resolves kinetic scale flow physics, the high dimension of the equation makes the computations expensive in practical 3D engineering applications. On the other hand, the hydrodynamic model, namely the Euler or Navier-Stokes (NS) equations, is mostly used in the continuum flow regime. For the plasma transport, the flow physics becomes more complicated. The plasma regime varies not only from rarefied to continuum regimes according to the Knudsen number, but also from the two-fluid to magnetohydrodynamic (MHD) regimes according to the normalized Larmor radius and Debye length. In the rarefied flow regime with large Knudsen number, the plasma physics is described by the kinetic Fokker-Planck-Landau equation coupled with the Maxwell equation [6]. In the hydrodynamic regime at small Knudsen number, the two-fluid hydrodynamic system coupled with Maxwell equation can describe the plasma dynamics in an effective way, which takes into account the Hall effect, electron inertia effect, and resistive effect, etc. [7]. In the highly magnetized flow regime where the normalized Larmor radius approaches zero and Debye length is on the order of the reciprocal of the speed of light, a single fluid ideal MHD can be used to describe the large scale plasma physics [8]. For both multiscale gas mixture and plasma transport, the hydrodynamic descriptions are more effective, but limited in the continuum flow regime; while the kinetic models capture kinetic scale physics, but have complex form and high dimensionality. Therefore, in order to capture flow physics in different regimes in a corresponding most efficient way, the construction of multiscale method for gas mixture and plasma transport is highly demanded.

In general, the numerical methods for gas mixture and plasma transport can be categorized into the deterministic and stochastic methods. The deterministic discrete ordinate method (DVM) has great advantages in the simulation of low speed and small temperature variation flow due to the absence of statistical noise [9]. In the past decade, many deterministic numerical methods have been developed for multi-species gas mixture [10-14], as well as plasma transport [15-18]. On the other hand, when dealing with the high speed and 3D flow, the stochastic particle method shows great advantages in terms of memory reduction and computation efficiency. The direct simulation Monte Carlo (DSMC) method has been extended to gas mixture and chemical reaction [19]. For the simulation of plasma transport, the particle-in-cell (PIC) method has been developed and widely applied in industry [20]. For the traditional DVM, DSMC, and PIC methods, the numerical cell size is usually required to be less than the particle mean free path and the 
Debye length, and the time step is required to be less than the mean particle collision time. The constraints on the cell size and time step limit the application regime of the traditional DVM, DSMC, and PIC methods and it becomes impossible to use them in the continuum flow regime. In order to remove these constraints and develop the most efficient methods in the corresponding regimes, the asymptotic preserving schemes have been proposed that attempt to preserve the flow dynamics in the collisionless and the Euler limiting regimes $[10,21]$.

The unified gas-kinetic scheme (UGKS) proposed by Xu et al. is a multiscale numerical method for the simulation of gas flow $[22,23]$. In the last decade, the UGKS has been well developed and extended to the multiscale transports, such as radiative transfer [24], plasma physics [16], gas-particle multiphase flow [25], neutron transport [26], etc. The two important ingredients of the UGKS are: firstly, the evolution of velocity distribution function is coupled with the evolution of the macroscopic flow variables; secondly, the numerical flux of the UGKS is constructed from the integral solution of the kinetic equation which takes into account the accumulating effect from particle free stream and collision up to a time step scale. The UGKS has been proved to be a second order unified preserving scheme that can accurately capture the NS solutions when the cell size and time step being much larger than the particle mean free path and mean collision time [27], the same as the traditional NS solvers for the direct discretization of the macroscopic equations. To further improve the efficiency of the UGKS in the simulation of high speed flow, the unified gas-kinetic wave-particle (UGKWP) method has been proposed and applied in the simulation of multiscale gas dynamics and photon transport [27-29]. The construction of the UGKWP method follows the same direct modeling methodology of the UGKS: the evolution of individual microscopic particle is coupled with the evolution of macroscopic flow variables, and the multiscale particle evolution is controlled by the same integral solution of the kinetic model equation. The purpose of this work is to extend the UGKWP method to the field of multiscale gas mixture and plasma simulations. The proposed UGKWP method shares the same multiscale property with the UGKS [16] and DUGKS $[17,18]$. The scheme preserves the collisionless limit in the rarefied regime, and the corresponding NS and MHD solvers in the comtinuum flow regime. An appealing feature of the UGKWP is that the computational complexity diminishes with the transition of the flow regime from kinetic to the continuum one. Especially, in the NS and MHD regime, the UGKWP converges to hydrodynamic flow solvers.

The rest of the paper is organized as follows. The governing equations for gas mixture and plasma transport are discussed in Section 2. In Section 3, the UGKWP methods for gas mixture and plasma transport are proposed. The unified preserving and asymptotic complexity diminishing properties of the UGKWP are discussed in Section 4. The numerical examples are shown in Section 5, and the last Section 6 is the conclusion.

\section{Governing equations for multi-species gas mixture and plasma transport}

This section is about the governing equations to be used in the construction of the schemes. The multi-species Boltzmann equation is first reviewed, and then the kinetic model equation proposed by Andries, et al. [3] will be discussed, including its asymptotic behavior in continuum regime. The two fluid kinetic-Maxwell system, as well as the Hall-MHD equations will be presented. 


\subsection{Multi-species Boltzmann equation}

A gas mixture composed of $m$ species can be modeled by the multi-species Boltzmann equations,

$$
\partial_{t} f_{\alpha}+\vec{v} \cdot \nabla f_{\alpha}=\sum_{k=1}^{m} \mathcal{Q}_{\alpha k}\left(f_{\alpha}, f_{k}\right),
$$

where $f_{\alpha}(t, \vec{x}, \vec{v})$ is the velocity distribution function of species $\alpha$, and the collision between species $\alpha$ and $k$ follows the integral operator

$$
\mathcal{Q}_{\alpha k}\left(f_{\alpha}, f_{k}\right)=\int_{\mathcal{R}^{3}} \int_{\mathcal{S}^{2}}\left(f_{\alpha}^{\prime} f_{k}^{*^{\prime}}-f_{\alpha} f_{k}^{*}\right) B^{\alpha, k}\left(\vec{v}_{r} \cdot \vec{n},\left|\vec{v}_{r}\right|\right) d \vec{n} d v^{*}
$$

where $f_{k}^{*}=f_{k}\left(t, \vec{x}, \vec{v}^{*}\right), f_{\alpha}^{\prime}=f_{\alpha}\left(t, \vec{x}, \vec{v}^{\prime}\right), f_{k}^{*^{\prime}}=f_{k}\left(t, \vec{x}, \vec{v}^{*^{\prime}}\right)$. The post collision velocity $\vec{v}$ and $\vec{v}^{*^{\prime}}$ follow

$$
\begin{aligned}
& \vec{v}^{\prime}=\vec{v}-2 \frac{\mu_{\alpha k}}{m_{\alpha}}\left(\vec{v}_{r} \cdot \vec{n}\right) \vec{n}, \\
& \vec{v}^{*^{\prime}}=\vec{v}^{*}+2 \frac{\mu_{\alpha k}}{m_{k}}\left(\vec{v}_{r} \cdot \vec{n}\right) \vec{n},
\end{aligned}
$$

where $\mu_{\alpha k}=\frac{m_{\alpha} m_{k}}{m_{\alpha}+m_{k}}$ is the reduced mass, and $\vec{n}$ is the unit vector joining the centers of the two colliding spheres. The collision kernel $B^{\alpha, k}$ depends on relative velocity $\vec{v}_{r}=\vec{v}-\vec{v}^{*}$. The macroscopic density $\rho_{\alpha}$, velocity $\vec{U}_{\alpha}$, temperature $T_{\alpha}$, and energy $E_{\alpha}$ of species $\alpha$ can be calculated by taking the moments of the velocity distribution $f_{\alpha}$,

$$
\begin{aligned}
& \rho_{\alpha}=m_{\alpha} n_{\alpha}=\int_{R^{3}} f_{\alpha} d \vec{v}, \quad \rho_{\alpha} \vec{U}_{\alpha}=\int_{R^{3}} \vec{v} f d \vec{v}, \\
& T_{\alpha}=\frac{1}{3 n_{\alpha} k_{B}} \int_{R^{3}}\left(\vec{v}-\vec{U}_{\alpha}\right)^{2} f d \vec{v}, \quad E_{\alpha}=\frac{1}{2} \rho_{\alpha}\left|\vec{U}_{\alpha}\right|^{2}+\frac{3}{2} n_{\alpha} k_{B} T_{\alpha},
\end{aligned}
$$

where $m_{\alpha}$ and $n_{\alpha}$ are the molecular mass and number density of species $\alpha$. For m species gas mixture, the total density $\rho$, total number density $n$, total momentum $\rho \vec{U}$, and total energy $E$ satisfy

$$
\begin{aligned}
& \rho=\sum_{\alpha=1}^{m} \rho_{\alpha}, \quad n=\sum_{\alpha=1}^{m} n_{\alpha}, \\
& \rho \vec{U}=\sum_{\alpha=1}^{m} \rho_{\alpha} \vec{U}_{\alpha}, \quad E=\sum_{\alpha=1}^{m} E_{\alpha} .
\end{aligned}
$$

Boltzmann equation is a fundamental equation that describes the mean free path level flow physics, however, to numerically solve the five-fold collision operator is costly. Simplified kinetic model equations are developed in the literature [30-33], including a relaxation-type kinetic model proposed by Andries, et al. [3]. AAP model will be introduced in the next section, based on which the numerical schemes for multi-species gas mixture and plasma are constructed.

\subsection{Kinetic model equation for multi-species gas mixture}

The relaxation-type kinetic model equation that originally proposed by Gross and Krook [34] has been widely used in the numerical simulation of rarefied gas dynamics due to its simple formulation. Such BGK-type operator has been extended to model the multispecies collision by Andries, Aoki, and Perthame [3], which can be written as

$$
\partial_{t} f_{\alpha}+\vec{v} \cdot \nabla_{x} f_{\alpha}=\frac{g_{\alpha}-f_{\alpha}}{\tau_{\alpha}},
$$


where the post collision distribution function is a Maxwellian distribuion

$$
g_{\alpha}=\rho_{\alpha}\left(\frac{m_{\alpha}}{2 \pi k T_{\alpha}^{*}}\right)^{3 / 2} \exp \left(-\frac{m_{\alpha}}{2 k_{B} T_{\alpha}^{*}}\left(\vec{v}-\vec{U}_{\alpha}^{*}\right)^{2}\right),
$$

and the parameters $T_{\alpha}^{*}$ and $\vec{U}_{\alpha}^{*}$ are chosen to recover the exchanging relations for Maxwell molecules,

$$
\begin{aligned}
& \vec{U}_{\alpha}^{*}=\vec{U}_{\alpha}+\frac{\tau_{\alpha}}{m_{\alpha}} \sum_{k=1}^{N} 2 \mu_{\alpha k} \chi_{\alpha k} n_{k}\left(\vec{U}_{k}-\vec{U}_{\alpha}\right) \\
& T_{\alpha}^{*}=T_{\alpha}-\frac{m_{\alpha}}{3 k_{B}}\left(\vec{U}_{\alpha}^{*}-\vec{U}_{\alpha}\right)^{2}+\frac{\tau_{\alpha}}{m_{\alpha}} \sum_{k=1}^{N} \frac{4 \mu_{\alpha} \chi_{\alpha k} n_{k}}{m_{\alpha}+m_{k}}\left(T_{k}-T_{\alpha}+\frac{m_{k}}{3 k_{B}}\left(\vec{U}_{k}-\vec{U}_{\alpha}\right)^{2}\right) .
\end{aligned}
$$

For Maxwell molecules, the interaction coefficient $\chi$ and relaxation parameter $\tau$ satisfy

$$
\frac{1}{\tau_{\alpha}}=\sum_{k=1}^{N} \chi_{\alpha k} n_{k}, \quad \chi_{\alpha k}=0.422 \pi\left(\frac{a_{\alpha k}\left(m_{\alpha}+m_{r}\right)}{m_{\alpha} m_{r}}\right)^{\frac{1}{2}},
$$

where $a_{\alpha k}$ is the constant of proportionality in the intermolecular force law [35]. The advantage of AAP model is that it satisfies the indifferentiability principle, entropy condition, and can recover the exchanging relationship of Maxwell molecules with such a simple relaxation form [3].

Based on AAP model, Liu et al. proposed a BGK-Maxwell system for fully ionized plasma transport [16], which can be written as

$$
\begin{aligned}
& \frac{\partial f_{\alpha}}{\partial t}+\vec{v} \cdot \nabla_{x} f_{\alpha}+\vec{a}_{\alpha} \cdot \nabla_{x} f_{\alpha}=\frac{g_{\alpha}-f_{\alpha}}{\tau_{\alpha}}, \\
& \frac{\partial \vec{B}}{\partial t}+\nabla_{x} \times \vec{E}=0, \\
& \frac{\partial \vec{E}}{\partial t}-\vec{c}^{2} \nabla_{x} \times \vec{B}=\frac{1}{\epsilon_{0}} \vec{j},
\end{aligned}
$$

where $\vec{j}=e\left(n_{i} \vec{U}_{i}-n_{e} \vec{U}_{e}\right)$ is the electric current, and the velocity distribution $f_{\alpha}(t, \vec{x}, \vec{v})$ of species $\alpha$ ( $\alpha=i$ for ion and $\alpha=e$ for electron) is governed by a kinetic equation that coupled with the Maxwell equations for electromagnetic wave. In the Maxwell equations, $\vec{E}$ and $\vec{B}$ are the electric and magnetic field, $\vec{c}$ is speed of light, and $\epsilon_{0}$ is the vacuum permittivity. In the kinetic equation, the Lorenz acceleration $\vec{a}_{s}$ takes the form

$$
\vec{a}_{s}=\frac{e(\vec{E}+\vec{v} \times \vec{B})}{m_{s}},
$$

where $e$ is electric charge, and $m_{s}$ is the particle mass of species $s$. The post collision distribution $g_{\alpha}$ takes the same form of the AAP model as given in Eq. (5), however the interspecies interaction coefficient $\chi_{i e}$ is determined by the plasma electrical conductivity $\sigma_{p}[16]$

$$
\chi_{i e} \sigma_{p}=\frac{n_{i} e\left(m_{i}+m_{e}\right)}{2 m_{i} m_{e}} .
$$

The hydrodynamic equations such as the Navier-Stokes equations, the Euler equations, and magnetohydrodynamic equations can be derived in the continuum regime. The asymptotic behavior of above kinetic model Eqs. (4) and (7) will be briefly discussed in the next subsection. 


\subsection{Asymptotic behavior of the kinetic system}

In this section, the asymptotic analysis is applied to give the corresponding hydrodynamic limits of the AAP model and BGK-Maxwell equations. Given the reference length $L_{\infty}$, temperature $T_{\infty}$, mass $m_{\infty}$, number density $n_{\infty}$, and magnetic field strength $B_{\infty}$, the following reference variables can be deduced,

$$
\begin{aligned}
& V_{\infty}=\sqrt{2 k_{B} T_{\infty} / m_{\infty}}, \quad t_{\infty}=L_{\infty} / V_{\infty}, \quad \rho_{\infty}=m_{\infty} n_{\infty} \\
& E_{\infty}=B_{\infty} V_{\infty}, \quad a_{\infty}=e B_{\infty} V_{\infty} / m_{\infty}, \quad f_{\infty}=m_{\infty} n_{\infty} / V_{\infty}^{3}
\end{aligned}
$$

which are the reference velocity, time, density, electric field, acceleration, and velocity distribution respectively. Based on the above reference variables, the AAP model can be re-scaled as

$$
\partial_{\tilde{t}} \tilde{f}_{\alpha}+\tilde{\vec{v}} \cdot \nabla_{\tilde{x}} \tilde{f}_{\alpha}=\frac{\tilde{g}_{\alpha}-\tilde{f}_{\alpha}}{\tilde{\tau}_{\alpha}},
$$

and the BGK-Maxwell system can be re-scaled as

$$
\begin{aligned}
& \frac{\partial \tilde{f}_{\alpha}}{\partial \tilde{t}}+\tilde{\vec{v}} \cdot \nabla_{\tilde{x}} \tilde{f}_{\alpha}+\frac{1}{\tilde{r}} \overrightarrow{\tilde{a}}_{\alpha} \cdot \nabla_{\tilde{x}} \tilde{f}_{\alpha}=\frac{\tilde{g}_{\alpha}-\tilde{f}_{\alpha}}{\tilde{\tau}_{\alpha}} \\
& \frac{\partial \tilde{\vec{B}}}{\partial \tilde{t}}+\nabla_{\tilde{x}} \times \tilde{\vec{E}}=0 \\
& \frac{\partial \tilde{\vec{E}}}{\partial \tilde{t}}-\tilde{c}^{2} \nabla_{\tilde{x}} \times \tilde{\vec{B}}=\frac{1}{\tilde{\lambda}_{D}^{2} \tilde{j}},
\end{aligned}
$$

where the variables with a tilde stand for the re-scaled variables, and especially $\tilde{r}$ and $\tilde{\lambda}_{D}$ are the normalized Larmor radius and Debye length,

$$
\tilde{r}=\frac{e B_{\infty} L_{\infty}}{m_{\infty} V_{\infty}}, \quad \tilde{\lambda}_{D}=\sqrt{\frac{\epsilon_{0} m_{\infty} V_{\infty}^{2}}{n e^{2}}} \frac{m_{\infty} V_{\infty}}{e B_{\infty}} .
$$

For the sake of simplicity, the tilde is omitted in the following parts of the paper.

In the continuum regime, the AAP model recovers the Navier-Stokes and Euler equations as $\tau \rightarrow 0$,

$$
\text { AAP model } \stackrel{\tau, \chi \ll 1}{\longrightarrow} \text { Navier-Stokes equations } \stackrel{\tau, \chi \rightarrow 0}{\longrightarrow} \text { Euler equations. }
$$

According to the Chapman-Enskog theory [1], the distribution of AAP model can be expanded as

$$
f_{\alpha}=g-\tau_{\alpha}\left(\partial_{t} \bar{g}_{\alpha}+\vec{v} \cdot \nabla_{x} \bar{g}_{\alpha}\right)+O\left(\tau^{2}\right)
$$

where $\bar{g}$ is the Maxwellian distribution of the averaged quantities of all species that are evaluated from Eq. (3). The zero-th order expansion with respect to $\tau_{\alpha}$ gives the Euler equations [3],

$$
\begin{aligned}
& \partial_{t} \rho_{\alpha}+\nabla \cdot\left(\rho_{\alpha} \vec{U}\right)=0, \\
& \partial_{t}(\rho \vec{U})+\nabla \cdot(p \mathbb{I}+\rho \vec{U} \vec{U})=0, \\
& \partial_{t} E+\nabla \cdot(E \vec{U}+p \vec{U})=0,
\end{aligned}
$$

and the first order expansion gives the Navier-Stokes equations [3],

$$
\begin{aligned}
& \partial_{t} \rho_{\alpha}+\nabla \cdot\left(\rho_{\alpha} \vec{U}+\vec{J}_{\alpha}\right)=0, \\
& \partial_{t}(\rho \vec{U})+\nabla \cdot(p \mathbb{I}+\rho \vec{U} \vec{U}+\sigma)=0, \\
& \partial_{t} E+\nabla \cdot(E \vec{U}+p \vec{U}+\sigma \cdot \vec{U}+\vec{q})=0 .
\end{aligned}
$$


The mass diffusion flux $\vec{J}_{\alpha}$ is

$$
\vec{J}_{\alpha}=-\sum_{k=1}^{m} L_{\alpha k} \frac{\nabla_{x}\left(n_{\alpha} k_{B} T\right)}{\rho_{\alpha}},
$$

and the shear stress $\sigma$ and heat flux $q$ satisfy

$$
\begin{aligned}
& \sigma=-\mu\left(\nabla_{x} \vec{U}+\left(\nabla_{x} \vec{U}\right)^{T}-\frac{2}{3} \nabla \cdot \vec{U} \mathbb{I}\right), \\
& \vec{q}=\frac{5}{2} k_{B} T \sum_{k=1}^{n} \frac{J_{k}}{m_{k}}-\kappa \nabla_{x} T,
\end{aligned}
$$

with the viscous coefficient $\mu=k_{B} T \sum_{k=1}^{m} \tau_{\alpha k} n_{k}$, and the heat conduction coefficient $\kappa=\frac{5}{2} k_{B}^{2} T \sum_{k=1}^{m} \frac{\tau_{k} n_{k}}{m_{k}}$. The Prandtl number of the AAP model is one, which means the model cannot preserve the correct relaxation of both stress and heat flux. To fix the Prandtl number, the ellipsoidal statistical model is proposed by Brull [4]. The mass flux coefficient matrix $L$ is a symmetric matrix [3]

$$
L=-(M-\kappa \mathbb{I})^{-1} \Omega,
$$

with

$$
\begin{array}{ll}
M_{i j}=-\sum_{k^{\prime} \neq i} \frac{2 \kappa_{i k}}{m_{i}+m_{k}} \rho_{k} & \text { if } \quad i=k \\
M_{i k}=A_{i k} \rho_{i} & \text { if } \quad i \neq k,
\end{array}
$$

and

$$
\Omega_{i k}=\rho_{i} \delta_{i k}-\frac{\rho_{i} \rho_{k}}{\rho} .
$$

In the mass flux, the Soret and the Dufour coefficients are equal to zero, which stand only for Maxwell particles. For more general potentials, this does not hold. Moreover, for Maxwell particles, the mass diffusion coefficient are not consistent with that derived from the Boltzamnn operator.

The BGK-Maxwell system converges to the two-fluid system and magnetohydrodynamics system as $\tau \rightarrow 0$ and $r \rightarrow 0$,

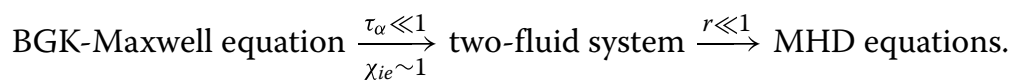

In the continuum regime with $\tau_{\alpha} \ll 1$ and $\chi_{i e} \sim 1$, the distribution of BGK-Maxwell system can be expanded as

$$
f_{\alpha}=g_{\alpha}-\tau_{\alpha}\left(\partial_{t} g_{\alpha}+\vec{v} \cdot \nabla_{x} g_{\alpha}\right)+O\left(\tau^{2}\right),
$$

where $g_{\alpha}$ is the Maxwellian distribution of the macroscopic quantities of species $\alpha$. The first order expansion gives hydrodynamic two-fluid equations

$$
\begin{aligned}
& \partial_{t} \rho_{\alpha}+\nabla_{x} \cdot\left(\rho_{\alpha} \vec{U}_{\alpha}\right)=0, \\
& \partial_{t}\left(\rho_{\alpha} \vec{U}_{\alpha}\right)+\nabla_{x} \cdot\left(\rho_{\alpha} \vec{U}_{\alpha} \vec{U}_{\alpha}+p_{\alpha} \mathbb{I}-\mu \sigma\left(\vec{U}_{\alpha}\right)\right)=\frac{n_{\alpha}}{r_{L_{i}}}\left(\vec{E}+\vec{U}_{\alpha} \times \vec{B}\right)+S_{\alpha}, \\
& \partial_{t} E_{\alpha}+\nabla_{x} \cdot\left(\left(E_{\alpha}+p_{\alpha}\right) \vec{U}_{\alpha}-\mu_{\alpha} \sigma\left(\vec{U}_{\alpha}\right) \vec{U}+\kappa_{\alpha} \nabla_{x} T\right)=\frac{n_{\alpha}}{r_{L_{i}}} \vec{U}_{\alpha} \cdot \vec{E}+Q_{\alpha},
\end{aligned}
$$

where the strain rate tensor $\sigma(\vec{U})$ is

$$
\sigma\left(\vec{U}_{\alpha}\right)=\left(\nabla_{x} \vec{U}_{\alpha}+\left(\nabla_{x} \vec{U}_{\alpha}\right)^{T}\right)-\frac{2}{3} \operatorname{div}_{x} \vec{U}_{\alpha} \mathbb{I} .
$$


The viscosity $\mu_{\alpha}$ and the thermal conductivity $\kappa_{\alpha}$ can be expressed by the relaxation parameter $\tau_{\alpha}$ as

$$
\mu_{\alpha}=\tau_{\alpha} n_{\alpha} k_{B} T_{\alpha}, \quad \kappa_{\alpha}=\tau_{\alpha} \frac{5}{2} \frac{k_{B}}{m} n k_{B} T .
$$

In above two-fluid system, $S_{i}=-S_{e}$ and $Q_{i}=-Q_{e}$ are the corresponding momentum and energy exchange between electron and ion,

$$
\begin{aligned}
S_{\alpha} & =\sum_{r} \frac{2 m_{\alpha} m_{r}}{m_{\alpha}+m_{r}} n_{\alpha} \chi_{\alpha r}\left(\vec{U}_{r}-\vec{U}_{\alpha}\right), \\
Q_{\alpha} & =\sum_{r} \frac{4 m_{\alpha} m_{r}}{\left(m_{\alpha}+m_{r}\right)^{2}} n_{r} \chi_{\alpha r}\left(\frac{3}{2} k_{B} T_{r}-\frac{3}{2} k_{B} T_{\alpha}+\frac{m_{r}}{2}\left(\vec{U}_{r}-\vec{U}_{\alpha}\right)^{2}\right) .
\end{aligned}
$$

In the magnetohydrodynamic regime with $m_{e} \ll m_{i}$, the first order with respect to $r$, the zero-th order with respect of $\tau_{\alpha}$ and $m_{e} / m_{i}$ of the two-fluid system give the Hall-MHD equations,

$$
\begin{aligned}
& \partial_{t} \rho+\nabla_{x} \cdot(\rho \vec{U})=0, \\
& \partial_{t}(\rho \vec{U})+\nabla_{x} \cdot(\rho \vec{U} \vec{U}+p \mathbb{I})=\lambda_{D}^{2} c^{2} \nabla_{x} \times \vec{B} \times \vec{B}, \\
& \partial_{t} E_{\alpha}+\nabla_{x} \cdot\left(\left(E_{\alpha}+p_{\alpha}\right) \vec{U}_{\alpha}\right)=\lambda_{D}^{2} c^{2} n \vec{U} \cdot\left(\nabla_{x} \times \vec{B} \times \vec{B}\right), \\
& \partial_{t} \vec{B}+\nabla_{x} \times \vec{E}=0, \\
& \vec{E}+\vec{U} \times \vec{B}=\underbrace{\frac{r}{\sigma} \vec{j}}_{\text {Resistive term }}+\underbrace{\frac{r}{n} \lambda_{D}^{2} c^{2} \nabla_{x} \times \vec{B} \times \vec{B},}_{\text {Hall term }}
\end{aligned}
$$

where $\vec{j}=e n_{i} \vec{U}_{i}-e n_{e} \vec{U}_{e}$ is the current density, and $\sigma=\frac{n_{i} e\left(m_{i}+m_{e}\right)}{2 m_{i} m_{e} \chi_{i e}}$ is the electrical conductivity. In the limit where $\lambda_{D}=c^{-1}$ and $r \rightarrow 0$, one gets the ideal MHD equations,

$$
\begin{aligned}
& \partial_{t} \rho+\nabla_{x} \cdot(\rho \vec{U})=0, \\
& \partial_{t}(\rho \vec{U})+\nabla_{x} \cdot(\rho \vec{U} \vec{U}+p I)=\nabla_{x} \times \vec{B} \times \vec{B}, \\
& \partial_{t} E+\nabla_{x} \cdot((E+p) \vec{U})=\vec{U} \cdot\left(\nabla_{x} \times \vec{B} \times \vec{B}\right), \\
& \partial_{t} \vec{B}+\nabla_{x} \times(\vec{U} \times \vec{B})=0 .
\end{aligned}
$$

The asymptotic limiting equations of the AAP model and BGK-Maxwell system are given in the above discussion. In the next section, the unified gas-kinetic wave-particle method for gas mixture and plasma transport will be proposed.

\section{Unified gas-kinetic wave-particle method}

\subsection{UGKWP method for multi-species gas mixture}

The unified gas-kinetic wave-particle method is a multiscale numerical method that preserves the asymptotic limits of the AAP model. The UGKWP method couples the evolution of the velocity distribution $f_{\alpha}$ and the macroscopic quantities $\vec{W}_{\alpha}$. The evolution of microscopic distribution and macroscopic variables will be given in the following subsections.

\subsubsection{The evolution of microscopic velocity distribution function}

Similar to the UGKWP method for single species gas [27], in current scheme the velocity distribution function is partially represented by an analytical distribution $g_{\alpha}^{+, c}$ and partially represented by stochastic particles $P_{\alpha k}=\left(m_{\alpha k}, \vec{x}_{\alpha k}, \vec{v}_{\alpha k}\right)$, which are shown in Fig. 1. 


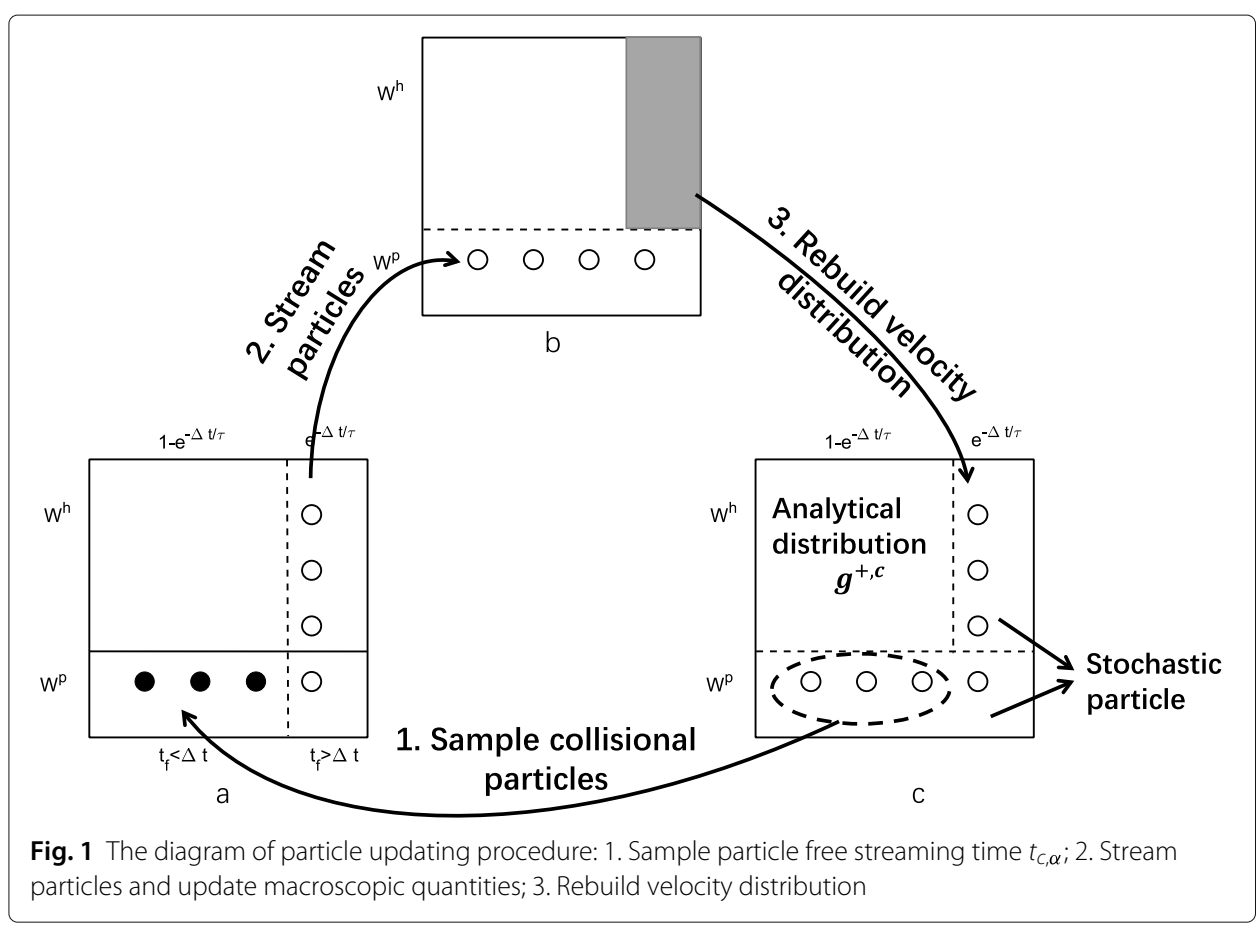

Here $m_{\alpha k}$ is the mass of simulation particle $P_{\alpha k}$, which represents a cluster of real gas particles of species $\alpha$, and $\vec{x}_{\alpha k}, \vec{v}_{\alpha k}$ stand for the position and velocity of simulation particle $P_{\alpha k}$. The evolution of the microscopic velocity distribution function follows the integral solution of the kinetic Eq. (4). With initial condition $f_{\alpha}(0, \vec{x}, \vec{v})=f_{\alpha, 0}(\vec{x}, \vec{v})$, the integral solution at $(\vec{x}, t)$ can be written as

$$
f_{\alpha}(\vec{x}, t, \vec{v})=\frac{1}{\tau} \int_{0}^{t} e^{-\left(t-t^{\prime}\right) / \tau} g_{\alpha}\left(\vec{x}^{\prime}, t^{\prime}, \vec{v}\right) d t^{\prime}+e^{-t / \tau} f_{\alpha, 0}\left(\vec{x}_{0}, \vec{v}\right),
$$

where the equilibrium distribution is integrated along the characteristics $\vec{x}^{\prime}=\vec{x}+\vec{v}\left(t^{\prime}-t\right)$. Substituting the second order Taylor expansion of equilibrium

$g_{\alpha}\left(\vec{x}^{\prime}, t^{\prime}, \vec{v}\right)=g_{\alpha}(\vec{x}, t, \vec{v})+\nabla_{x} g_{\alpha}(\vec{x}, t, \vec{v}) \cdot\left(\vec{x}^{\prime}-\vec{x}\right)+\partial_{t} g_{\alpha}(\vec{x}, t, \vec{v})\left(t^{\prime}-t\right)+O\left(\left(\vec{x}^{\prime}-\vec{x}\right)^{2},\left(t^{\prime}-t\right)^{2}\right)$, into the integral solution, the numerical multiscale evolution solution for simulation particle can be obtained,

$$
f_{\alpha}(\vec{x}, t, \vec{v})=\left(1-e^{-t / \tau}\right) g_{\alpha}^{+}(\vec{x}, t, \vec{v})+e^{-t / \tau} f_{0, \alpha}\left(\vec{x}_{0}, \vec{v}\right),
$$

where

$$
g_{\alpha}^{+}(\vec{x}, t, \vec{v})=g_{\alpha}(\vec{x}, t, \vec{v})+\left(\frac{t e^{-t / \tau}}{1-e^{-t / \tau}}-\tau\right)\left(\partial_{t} g_{\alpha}(\vec{x}, t, \vec{v})+\vec{v} \cdot \nabla_{x} g_{\alpha}(\vec{x}, t, \vec{v})\right) .
$$

A physical interpretation of Eq. (20) is that a particle has a probability $e^{-t / \tau}$ to free stream in a time period $[0, t]$, and has a probability $\left(1-e^{-t / \tau}\right)$ to interact with other particles and reach a velocity distribution $g_{\alpha}^{+}$. The free stream particles are kept and the collisional particles get re-sampled from distribution $g_{\alpha}^{+}$. The cumulative distribution function of the free streaming time $t_{f}$ is

$$
F\left(t_{f}<t\right)=\exp (-t / \tau)
$$


from which $t_{f}$ can be sampled as $t_{f}=-\tau \ln (\eta)$ with $\eta$ a uniform distribution $\eta \sim U(0,1)$. For a time step $\Delta t$, the particles with $t_{f} \geq \Delta t$ will be collisionless particles, and the particles with $t_{f}<\Delta t$ will be collisional particles. The procedure of updating particles in UGKWP method is

Step 1: Sample free streaming time $t_{f, \alpha k}$ for each particle $P_{\alpha k}$, and stream particle $P_{\alpha k}$ for a time period of $\min \left(\Delta t, t_{f, \alpha k}\right)$;

Step 2: Keep collisionless particles, and remove collisional particles. Calculate the total conservative quantities of collisional particles $\vec{W}_{i, \alpha}^{h}$ from the updated conservative quantities $\vec{W}_{i, \alpha}$ as $\vec{W}_{i, \alpha}^{h}=\vec{W}_{i, \alpha}-\vec{W}_{i, \alpha}^{p}$;

Step 3: Rebuild the microscopic velocity distribution. Calculate the analytical distribution $g_{\alpha}^{+, c}$ and re-sample collisional particles from distribution $g_{\alpha}^{+, f}$.

In the above particle updating procedure, the total conservative quantities of collisionless particles in cell $\Omega_{i}$ are denoted as $\vec{W}_{i, \alpha}^{p}$, and the total conservative quantities of collisional particles in cell $\Omega_{i}$ are denoted as $\vec{W}_{i, \alpha}^{h}$. In the distribution rebuilding process, the $\vec{W}_{i, \alpha}^{h}$ is divided into $g_{\alpha}^{+, c}=\left(1-e^{-\Delta t / \tau^{n+1}}\right) g_{\alpha}^{+}$and $g_{\alpha}^{+, f}=e^{-\Delta t / \tau^{n+1}} g_{\alpha}^{+}$, corresponding to the collisional and collisionless particles in the next time step from $t^{n}$ to $t^{n+1}$. The distribution $g_{\alpha}^{+, c}$ is recorded analytically, and the distribution $g_{\alpha}^{+, f}$ is re-sampled into stachastic particles. Above discussion gives the evolution of particles, and in the next subsection we will give the evolution of the conservative variables.

\subsubsection{The evolution of macroscopic quantities}

The evolution of macroscopic quantities is under the framework of finite volume scheme. The cell averaged conservative variables $\vec{W}_{i, \alpha}=\left(\rho_{i, \alpha}, \rho_{i, \alpha} \vec{U}_{i, \alpha}, \rho_{i, \alpha} E_{i, \alpha}\right)$ on a physical cell $\Omega_{i}$ are defined as

$$
\vec{W}_{i, \alpha}=\frac{1}{\left|\Omega_{i}\right|} \int_{\Omega_{i}} \vec{W}_{\alpha}(\vec{x}) d \vec{x} .
$$

The finite volume scheme of $\vec{W}_{i, \alpha}$ follows

$$
\vec{W}_{i, \alpha}^{n+1}=\vec{W}_{i, \alpha}^{n}-\sum_{s} \frac{\Delta t}{\left|\Omega_{i}\right|}\left|l_{s}\right| F_{s, \alpha}+\frac{\Delta t}{\tau}\left(\vec{W}_{i, \alpha}^{* n+1}-\vec{W}_{i, \alpha}^{n+1}\right),
$$

where $l_{s} \in \partial \Omega_{i}$ is the cell interface with center $\vec{x}_{s}$ and outer unit normal vector $\vec{n}_{s}$. And $\vec{W}_{i, \alpha}^{* n+1}=\left(\rho_{i, \alpha}^{n+1}, \rho_{i, \alpha}^{n+1} \vec{U}_{i, \alpha}^{* n+1}, \rho_{i, \alpha}^{n+1} E_{i, \alpha}^{* n+1}\right)$ are calculated from Eq. (6). The numerical flux of conservative variables $F_{s, \alpha}$ at $\vec{x}_{s}$ can be written as

$$
F_{s, \alpha}=\frac{1}{\Delta t} \int_{t^{n}}^{t_{n+1}} \int \vec{v} \cdot \vec{n}_{s} f_{\alpha}\left(\vec{x}_{s}, t, \vec{v}\right) \vec{\Psi} d \Xi d t,
$$

where $\vec{\Psi}=\left(1, \vec{v}, \frac{1}{2}\left(\vec{v}^{2}+\xi^{2}\right)\right)$ is the conservative moments of distribution function with $\xi$ the internal degree of freedom. The time dependent distribution function $f_{\alpha}\left(\vec{x}_{s}, t, \vec{v}\right)$ at cell interface is constructed from the integral solution of kinetic equation as given in Eq. (19). The above UGKWP flux for conservative variables can be split into the equilibrium flux

$$
F_{s, \alpha}^{g}=\frac{1}{\Delta t} \int_{t^{n}}^{t_{n+1}} \int \vec{v} \cdot \vec{n}_{s}\left\{\frac{1}{\tau} \int_{0}^{t} e^{-\left(t-t^{\prime}\right) / \tau} g_{\alpha}\left(\vec{x}^{\prime}, t^{\prime}, \vec{v}\right) d t^{\prime}\right\} \vec{\Psi} d \Xi d t,
$$

and the free streaming flux

$$
F_{s, \alpha}^{f}=\frac{1}{\Delta t} \int_{t^{n}}^{t_{n+1}} \int \vec{v} \cdot \vec{n}_{s} e^{-t / \tau} f_{\alpha, 0}\left(\overrightarrow{x_{0}}, \vec{v}\right) \vec{\Psi} d \Xi d t .
$$


First, the equilibrium flux $F_{s}^{e q}$ can be calculated directly from the macroscopic flow field. Assume $\vec{x}_{s}=0$ and $t^{n}=0$, the equilibrium $g$ can be expanded as

$$
g_{\alpha}(\vec{x}, t, \vec{v})=g_{0, \alpha}+\nabla_{x} g_{0, \alpha} \cdot \vec{x}+\partial_{t} g_{0, \alpha} t,
$$

where $g_{0, \alpha}=g_{\alpha}(0,0, \vec{v})$. The initial equilibrium $g_{0, \alpha}$ and its spatial and time derivatives can be obtained from the micro-macro consistency

$$
\int g_{\alpha} \vec{\Psi} d \Xi=\int_{\vec{v} \cdot \vec{n}>0} g_{\alpha}^{l} \vec{\Psi} d \Xi+\int_{\vec{v} \cdot \vec{n}<0} g_{\alpha}^{r} \vec{\Psi} d \Xi, \quad \int \nabla_{x} g_{\alpha} \vec{\Psi} d \Xi=\nabla_{x} \vec{W}_{\alpha}
$$

and compatible condition

$$
\int \partial_{t} g_{\alpha} \vec{\Psi} d \Xi=-\int \vec{v} \cdot \nabla_{x} g_{\alpha} \vec{\Psi} d \Xi
$$

where $g_{\alpha}^{l}$ and $g_{\alpha}^{r}$ are the equilibrium distributions according to the reconstructed left and right side conservative variables at cell interface $\vec{W}_{\alpha}^{l}, \vec{W}_{\alpha}^{r}$, and $\nabla_{x} \vec{W}_{\alpha}$ is the reconstructed spatial derivative of conservative variables at cell interface. In this paper, the van Leer limiter is used to achieve a second order accuracy in space reconstruction. Substituting the reconstructed equilibrium distribution Eq. (26) into the equilibrium flux Eq. (24), we have

$$
F_{s, \alpha}^{g}=\int \vec{v} \cdot \vec{n}_{s}\left(C_{1} g_{0, \alpha}+C_{2} \vec{v} \cdot \nabla_{x} g_{0, \alpha}+C_{3} \partial_{t} g_{0, \alpha}\right) \vec{\Psi} d \Xi,
$$

where the time integration coefficients are

$$
\begin{aligned}
& C_{1}=1-\frac{\tau_{\alpha}}{\Delta t}\left(1-e^{-\Delta t / \tau_{\alpha}}\right), \\
& C_{2}=-\tau_{\alpha}+\frac{2 \tau_{\alpha}^{2}}{\Delta t}-e^{-\Delta t / \tau_{\alpha}}\left(\frac{2 \tau_{\alpha}^{2}}{\Delta t}+\tau_{\alpha}\right), \\
& C_{3}=\frac{1}{2} \Delta t-\tau_{\alpha}+\frac{\tau_{\alpha}^{2}}{\Delta t}\left(1-e^{-\Delta t / \tau_{\alpha}}\right) .
\end{aligned}
$$

Next we consider the free stream flux $F_{s, \alpha}^{f}$. As stated in the last subsection, the initial distribution is represented partially by an analytical distribution $g_{\alpha}^{+, c}$, and partially by particles, and therefore the free stream flux $F_{s, \alpha}^{f}$ is also calculated partially from the reconstructed analytical distribution as $F_{s, \alpha}^{f, w}$, and partially from particles as $F_{s, \alpha}^{f, p}$. The initial analytical distribution $g_{\alpha}^{+, c}$ is reconstructed as

$$
g_{0, \alpha}^{+, c}(\vec{x}, \vec{v})=g_{0, \alpha}^{+, c}+\nabla_{x} g_{0, \alpha}^{+, c} \cdot \vec{x},
$$

which gives

$$
F_{s, \alpha}^{f, w}=\int \vec{v} \cdot \vec{n}\left(C_{4} g_{0, \alpha}^{+}+C_{5} \vec{v} \cdot \nabla_{x} g_{0, \alpha}^{+}\right) \vec{\Psi} d \Xi,
$$

where the time integration coefficients are

$$
\begin{aligned}
& C_{4}=\frac{\tau_{\alpha}}{\Delta t}\left(1-e^{-\Delta t / \tau_{\alpha}}\right)-\Delta t e^{-\Delta t / \tau_{\alpha}}, \\
& C_{5}=\tau e^{-\Delta t / \tau_{\alpha}}-\frac{\tau_{\alpha}^{2}}{\Delta t}\left(1-e^{-\Delta t / \tau_{\alpha}}\right)+\frac{\Delta t^{2}}{2} e^{-\Delta t / \tau_{\alpha}} .
\end{aligned}
$$

The net particle flux $F_{s, \alpha}^{f, p}$ is calculated as

$$
F_{s, \alpha}^{f, p}=\frac{1}{\Delta t}\left(\sum_{k \in P_{\partial \Omega_{i}^{+}}, \alpha} \vec{W}_{P_{k, \alpha}}-\sum_{k \in P_{\partial \Omega_{i}^{-}, \alpha}} \vec{W}_{P_{k, \alpha}}\right),
$$


where $\vec{W}_{P_{k, \alpha}}=\left(m_{k, \alpha}, m_{k, \alpha} \vec{v}_{k, \alpha}, \frac{1}{2} m_{k, \alpha} \vec{v}_{k, \alpha}^{2}\right), P_{\partial \Omega_{i}^{-}, \alpha}$ is the index set of the particles streaming out of cell $\Omega_{i}$ during a time step, and $P_{\partial \Omega_{i}^{+}, \alpha}$ is the index set of the particles streaming into cell $\Omega_{i}$. Finally, the finite volume scheme for conservative variables is

$$
\vec{W}_{i, \alpha}^{n+1}=\vec{W}_{i, \alpha}^{n}-\sum_{s} \frac{\Delta t}{\left|\Omega_{i}\right|}\left|l_{s}\right| F_{s, \alpha}^{e q}-\sum_{s} \frac{\Delta t}{\left|\Omega_{i}\right|}\left|l_{s}\right| F_{s, \alpha}^{f r, w}+\frac{\Delta t}{\left|\Omega_{i}\right|} F_{s, \alpha}^{f, p}+\frac{\Delta t}{\tau}\left(\vec{W}_{i, \alpha}^{* n+1}-\vec{W}_{i, \alpha}^{n+1}\right) .
$$

To solve $\vec{W}_{i, \alpha}^{n+1}$ from Eq. (30), the following two linear system needs to be solved. The first is the $m \times m$ linear system for $m$ species velocity vector $\vec{V}_{i}^{n+1}=\left(\vec{U}_{i, 1}^{n+1}, \vec{U}_{i, 2}^{n+1}, \ldots, \vec{U}_{i, m}^{n+1}\right)$,

$$
A_{i} \vec{V}_{i}^{n+1}=B_{i},
$$

where $B_{\alpha, i}=\rho_{i, \alpha}^{n} \vec{U}_{i, \alpha}^{n}-\sum_{s} \frac{\Delta t}{\left|\Omega_{i}\right|}\left|l_{s}\right| F_{s, \alpha}^{\rho u}$, and $F_{s, \alpha}^{\rho u}$ is the momentum flux of $\sum_{s} \frac{\Delta t}{\left|\Omega_{i}\right|}\left|l_{s}\right| F_{s, \alpha}^{e q}+$ $\sum_{s} \frac{\Delta t}{\left|\Omega_{i}\right|}\left|l_{s}\right| F_{s, \alpha}^{f r, w}+\frac{\Delta t}{\left|\Omega_{i}\right|} F_{s, \alpha}^{f, p}$. The matrix $A_{i}$ read

$$
\begin{aligned}
& \left(A_{i}\right)_{\alpha \alpha}=\rho_{i, \alpha}^{n+1}+2 \Delta t \sum_{\substack{\beta=1 \\
\beta \neq \alpha}}^{m} \mu_{\alpha \beta} \kappa_{\alpha \beta} n_{i, \beta}^{n+1} \\
& \left(A_{i}\right)_{\alpha \beta}=-2 \Delta t \mu_{\alpha \beta} \kappa_{\alpha \beta} n_{i, \beta}^{n+1} .
\end{aligned}
$$

The second $m \times m$ linear system is for $m$ species internal energies $\vec{e}_{i}^{n+1}=$ $\left(e_{i, 1}^{n+1}, e_{i, 2}^{n+1}, \ldots e_{i, m}^{n+1}\right)$

$$
C_{i} \vec{e}_{i}^{n+1}=D_{i}
$$

where

$$
\begin{aligned}
D_{i, \alpha}= & E_{i, \alpha}^{n}-\sum_{s} \frac{\Delta t}{\left|\Omega_{i}\right|}\left|l_{s}\right| F_{s, \alpha}^{E}-\frac{1}{2} \rho_{i, \alpha}^{n+1}\left(\vec{U}_{i, \alpha}^{n+1}\right)^{2}+\frac{\Delta t \rho_{i, \alpha}^{n+1}}{2 \tau_{i, \alpha}^{n+1}}\left(\left(\vec{U}_{i, \alpha}^{* n+1}\right)^{2}-\left(\vec{U}_{i, \alpha}^{n+1}\right)^{2}\right) \\
& -\frac{\Delta t \rho_{i, \alpha}^{n+1}}{2 \tau_{i, \alpha}^{n+1}}\left(\vec{U}_{i, \alpha}^{* n+1}-\vec{U}_{i, \alpha}^{n+1}\right)^{2}+\Delta t n_{i, \alpha}^{n+1} \sum_{\substack{\beta=1 \\
\beta \neq \alpha}}^{m} \mu_{\alpha \beta} \kappa_{\alpha \beta} \frac{2 \rho_{i, \beta}^{n+1}}{m_{\alpha}+m_{\beta}}\left(\vec{U}_{i, \alpha}^{n+1}-\vec{U}_{i, \alpha}^{n+1}\right)^{2},
\end{aligned}
$$

with $F_{s, \alpha}^{E}$ is the energy flux of $\sum_{s} \frac{\Delta t}{\left|\Omega_{i}\right|}\left|l_{s}\right| F_{s, \alpha}^{e q}+\sum_{s} \frac{\Delta t}{\left|\Omega_{i}\right|}\left|l_{s}\right| F_{s, \alpha}^{f r, w}+\frac{\Delta t}{\left|\Omega_{i}\right|} F_{s, \alpha}^{f, p}$, and

$$
\begin{aligned}
\left(C_{i}\right)_{\alpha \alpha} & =n_{i, \alpha}^{n+1}+\Delta t n_{i, \alpha}^{n+1} \sum_{\substack{\beta=1 \\
\beta \neq \alpha}}^{m} \mu_{\alpha \beta} \kappa_{\alpha \beta} \frac{4 n_{i, \beta}^{n+1}}{m_{\alpha}+m_{\beta}}, \\
\left(C_{i}\right)_{\alpha \beta} & =-\Delta t n_{i, \alpha}^{n+1} \mu_{\alpha \beta} \kappa_{\alpha \beta} \frac{4 n_{i, \beta}^{n+1}}{m_{\alpha}+m_{\beta}} .
\end{aligned}
$$

Under the assumption of non-vacuum solutions $\left(\rho_{i, \alpha}^{n}>0\right)$, each system admits a unique solution. The evolution of the microscopic velocity distribution and macroscopic quantities compose the UGKWP method for multi-species gas mixture.

\subsection{UGKWP method for plasma transport}

In this subsection, the UGKWP method for plasma transport will be proposed, which is the UGKWP method for multi-species coupled with the electromagnetic field. We split the BGK-Maxwell equations into the transport equations and the interaction equations. 
The transport equations including the electron ion transport and electromagnetic wave transport can be written as

$$
\begin{aligned}
& \partial_{t} f_{\alpha}+\vec{v} \cdot \nabla_{x} f_{\alpha}=\frac{g_{\alpha}-f_{\alpha}}{\tau_{\alpha}}, \\
& \frac{\partial \vec{B}}{\partial t}+\nabla_{x} \times \vec{E}=0, \\
& \frac{\partial \vec{E}}{\partial t}-c^{2} \nabla_{x} \times \vec{B}=0,
\end{aligned}
$$

and the interaction equations are

$$
\begin{aligned}
& \partial_{t} f_{\alpha}+\frac{e_{\alpha}}{m_{\alpha} r}(\vec{E}+\vec{v} \times \vec{B}) \cdot \nabla_{v} f_{\alpha}=0, \\
& \frac{\partial \vec{E}}{\partial t}=-\frac{1}{\hat{\lambda}_{D}^{2} r} \vec{j} .
\end{aligned}
$$

In the next two subsections, the numerical evolution equations for the transport equations and interaction equations will be presented respectively.

\subsubsection{Evolution equations for the transport equations}

In the transport equations, the electron and ion transports are decoupled from the electromagnetic wave transport. The numerical evolution equation for the electron and ion transport is the UGKWP method presented in Section 3. The Yee-grid based Crank-Nicolson scheme proposed by Yang el al. is used as the evolution equation for the electromagnetic wave transport [36]. The semi-implicit discretization of transverse electric wave equation on Yee mesh can be written as

$$
\begin{aligned}
E_{x}^{n+1}\left(x_{i+\frac{1}{2}}, y_{j}\right)= & E_{x}^{n}\left(x_{i+\frac{1}{2}}, y_{j}\right)+\frac{\Delta t c^{2}}{2 \Delta y}\left(B_{z}^{n+1}\left(x_{i+\frac{1}{2}}, y_{j+\frac{1}{2}}\right)-B_{z}^{n+1}\left(x_{i+\frac{1}{2}}, y_{j-\frac{1}{2}}\right)\right) \\
& +\frac{\Delta t c^{2}}{2 \Delta y}\left(B_{z}^{n}\left(x_{i+\frac{1}{2}}, y_{j+\frac{1}{2}}\right)-B_{z}^{n}\left(x_{i+\frac{1}{2}}, y_{j-\frac{1}{2}}\right)\right), \\
E_{y}^{n+1}\left(x_{i}, y_{i+\frac{1}{2}}\right)= & E_{x}^{n}\left(x_{i}, y_{j+\frac{1}{2}}\right)-\frac{\Delta t c^{2}}{2 \Delta x}\left(B_{z}^{n+1}\left(x_{i+\frac{1}{2}}, y_{j+\frac{1}{2}}\right)-B_{z}^{n+1}\left(x_{i-\frac{1}{2}}, y_{j+\frac{1}{2}}\right)\right) \\
& -\frac{\Delta t c^{2}}{2 \Delta x}\left(B_{z}^{n}\left(x_{i+\frac{1}{2}}, y_{j+\frac{1}{2}}\right)-B_{z}^{n}\left(x_{i-\frac{1}{2}}, y_{j+\frac{1}{2}}\right)\right) .
\end{aligned}
$$

And the semi-implicit discretization of magnetic wave equation is

$$
\begin{aligned}
B_{z}^{n+1}\left(x_{i+\frac{1}{2}}, y_{i+\frac{1}{2}}\right)= & B_{z}^{n+1}\left(x_{i+\frac{1}{2}}, y_{i+\frac{1}{2}}\right)+\frac{\Delta t}{2 \Delta y}\left(E_{x}^{n+1}\left(x_{i+\frac{1}{2}}, y_{j+1}\right)-E_{x}^{n+1}\left(x_{i+\frac{1}{2}}, y_{j}\right)\right) \\
& +\frac{\Delta t}{2 \Delta y}\left(E_{x}^{n}\left(x_{i+\frac{1}{2}}, y_{j+1}\right)-E_{x}^{n}\left(x_{i+\frac{1}{2}}, y_{j}\right)\right) \\
& -\frac{\Delta t}{2 \Delta x}\left(E_{y}^{n+1}\left(x_{i+1}, y_{j+\frac{1}{2}}\right)-E_{y}^{n+1}\left(x_{i}, y_{j+\frac{1}{2}}\right)\right) \\
& +\frac{\Delta t}{2 \Delta x}\left(E_{y}^{n}\left(x_{i+1}, y_{j+\frac{1}{2}}\right)-E_{y}^{n}\left(x_{i}, y_{j+\frac{1}{2}}\right)\right)
\end{aligned}
$$


Substituting Eqs. (32) and (33) into Eq. (34), an implicit equation for $B_{z}$ can be derived as

$$
\begin{aligned}
& {\left[1-\frac{c^{2} \Delta t^{2}}{4}\left(D_{2 x}+D_{2 y}\right)\right] B_{z}^{n+1}\left(x_{i+\frac{1}{2}}, y_{j+\frac{1}{2}}\right)=\left[1+\frac{c^{2} \Delta t^{2}}{4}\left(D_{2 x}+D_{2 y}\right)\right] B_{z}^{n}\left(x_{i+\frac{1}{2}}, y_{j+\frac{1}{2}}\right)} \\
& \quad+f\left(E_{x}^{n}, E_{y}^{n}\right),
\end{aligned}
$$

which can be effectively solved by Douglas-Gunn algorithm. The advantage of the Yeegrid based Crank-Nicolson scheme is that the divergence constraint of the Maxwell equation is numerically preserved; the dispersion and dissipation error is lower than the FDTD method; and the scheme is unconditionally stable, which removes the CFL constraint on time step.

\subsubsection{Evolution equations for the interaction equations}

Taking conservative moments on Eq. (31), one gets the macroscopic interaction equations

$$
\left\{\begin{array}{l}
\frac{\rho_{\alpha} \vec{U}}{\partial t}=\frac{e_{\alpha} n_{\alpha}}{r}(\vec{E}+\vec{U} \times \vec{B}), \\
\frac{\partial \vec{E}}{\partial t}=-\frac{1}{\hat{\lambda}_{D}^{2} r} \vec{j}
\end{array}\right.
$$

The implicit discretization of the macroscopic interaction equations gives the following linear system,

$$
\left\{\begin{array}{l}
\rho_{i}^{n+1} \vec{U}_{i}^{n+1}-\rho_{i}^{n+1} \vec{U}_{i}^{n}=\frac{\Delta t}{r} n_{i}^{n+1}\left(\vec{E}^{n+1}+\vec{U}_{i}^{n+1} \times \vec{B}^{n+1}\right), \\
\rho_{e}^{n+1} \vec{U}_{e}^{n+1}-\rho_{e}^{n+1} \vec{U}_{e}^{n}=-\frac{\Delta t}{r} n_{e}^{n+1}\left(\vec{E}^{n+1}+\vec{U}_{e}^{n+1} \times \vec{B}^{n+1}\right), \\
\vec{E}^{n+1}-\vec{E}^{n}=-\frac{\Delta t}{\lambda_{D}^{2} r}\left(\vec{j}_{i}^{n+1}+\vec{j}_{e}^{n+1}\right),
\end{array}\right.
$$

from which the electromagnetic field and macroscopic flow variables are updated to $t^{n+1}$, and the velocity of the simulation particles is updated by

$$
\vec{v}_{k, \alpha}^{n+1}=\vec{v}_{k, \alpha}^{n}+\frac{\Delta t e_{\alpha}}{m_{\alpha} r}\left(\vec{E}^{n+1}+\vec{v}_{k, \alpha} \times \vec{B}^{n+1}\right) .
$$

The evolutions of the transport equations and interaction equations compose the UGKWP method for plasma transport.

\section{Analysis and discussion}

\subsection{Unified preserving and asymptotic complexity diminishing properties of UGKWP method}

In this section, the multiscale property of the UGKWP method will be discussed, and the computational complexity will be estimated. Guo et al. proposes the unified preserving property which assesses the order of accuracy of a kinetic scheme in continuum regime [37]. Crestetto et al. proposes the asymptotic complexity diminishing property of a kinetic scheme which assesses the computational complexity of a kinetic scheme in continuum regime [38]. In the following proposition, we show that the UGKWP method is a second order UP scheme and an asymptotic complexity diminishing scheme.

Proposition 4.1 Holding the mesh size and time step, the UGKWP method satisfies:

1 The scheme is consistent to the collisionless Boltzmann equation as the local relaxation parameter $\tau \rightarrow \infty$. 
2 The scheme becomes a second order scheme for the Navier-Stokes equations $\tau \rightarrow 0$.

3 The total degree of freedom of the scheme $N_{f} \rightarrow N_{f}^{h}$ as $\tau \rightarrow 0$, where $N_{f}^{h}$ is the freedom of the hydrodynamic equations.

Proof 1 In the collisionless limit, we have

$$
\lim _{\tau \rightarrow \infty} t_{f, \alpha}=\lim _{\tau \rightarrow \infty}(-\tau \ln (\eta)) \rightarrow \infty .
$$

Therefore, all particles will be streamed for $\min \left(\Delta t, t_{f, \alpha}\right)=\Delta t$. And the UGKWP method solves collisionless Boltzmann equation in collisionless regime.

2 In the continuum regime when $\tau \rightarrow 0$, we have

$$
\begin{aligned}
g_{\alpha}^{+}(\vec{x}, t, \vec{v}) & =g_{\alpha}(\vec{x}, t, \vec{v})+\left(\frac{t e^{-t / \tau}}{1-e^{-t / \tau}}-\tau\right)\left(\partial_{t} g_{\alpha}(\vec{x}, t, \vec{v})+\vec{v} \cdot \nabla_{x} g_{\alpha}(\vec{x}, t, \vec{v})\right) \\
& =g_{\alpha}(\vec{x}, t, \vec{v})-\tau\left(\partial_{t} g_{\alpha}(\vec{x}, t, \vec{v})+\vec{v} \cdot \nabla_{x} g_{\alpha}(\vec{x}, t, \vec{v})\right)+O\left(e^{-\Delta t / \tau}\right) \\
& =g_{\alpha}(\vec{x}, t, \vec{v})-\tau\left(\partial_{t} \bar{g}(\vec{x}, t, \vec{v})+\vec{v} \cdot \nabla_{x} \bar{g}(\vec{x}, t, \vec{v})\right)+O\left(\tau^{2}\right)
\end{aligned}
$$

The analytic flux $F_{\alpha}^{a n}$ of macroscopic variables, namely the equilibrium flux and free streaming flux by analytic distribution function satisfies

$$
\begin{aligned}
F_{\alpha}^{a n} & =F_{\alpha}^{e q}+F_{\alpha}^{f, w} \\
& =\int \vec{v} \cdot \vec{n}\left(C_{1} g_{0, \alpha}+C_{2} \vec{v} \cdot \nabla_{x} g_{0, \alpha}+C_{3} \partial_{t} g_{0, \alpha}\right) \vec{\Psi} d \Xi+\int \vec{v} \cdot \vec{n}\left(C_{4} g_{\alpha}^{+}+C_{5} \vec{v} \cdot \nabla_{x} g_{\alpha}^{+}\right) \vec{\Psi} d \Xi \\
& =\int \vec{v} \cdot \vec{n}\left(\left(C_{1}+C_{4}\right) g_{0, \alpha}+\left(C_{2}-\tau C_{4}+C_{5}\right) \vec{v} \cdot \nabla_{x} g_{0, \alpha}+\left(C_{3}-\tau C_{4}\right) \partial_{t} g_{0, \alpha}\right) \vec{\Psi} d \Xi \\
& =\int \vec{v} \cdot \vec{n}\left(g_{0, \alpha}-\tau \vec{v} \cdot \nabla_{x} g_{0, \alpha}+\left(\frac{1}{2} \Delta t-\tau\right) \partial_{t} g_{0, \alpha}\right) \vec{\Psi} d \Xi+O\left(e^{-\Delta t / \tau}\right) \\
& =\int \vec{v} \cdot \vec{n}\left(g_{0, \alpha}-\tau \vec{v} \cdot \nabla_{x} \bar{g}_{0}+\left(\frac{1}{2} \Delta t-\tau\right) \partial_{t} \bar{g}_{0}\right) \vec{\Psi} d \Xi+O\left(\tau^{2}\right)
\end{aligned}
$$

The sampled particle mass in the UGKWP method is $e^{-\Delta t / \tau} \rho_{\alpha}^{h} \Omega_{x}$ and therefore the net free streaming flow contributed by particles passing through the cell interface, $F_{s, \alpha}^{f, p} \sim O\left(e^{-\Delta t / \tau}\right)$, diminishes. As $\tau \rightarrow 0$, Eq. (30) exponentially converges to

$$
\begin{aligned}
\vec{W}_{i, \alpha}^{n+1}= & \vec{W}_{i, \alpha}^{n}-\sum_{s} \frac{\Delta t}{\left|\Omega_{i}\right|}\left|l_{s}\right| \int \vec{v} \cdot \vec{n}\left(g_{0 s, \alpha}-\tau\left(\partial_{t} \bar{g}_{0, s}+\vec{v} \cdot \nabla_{x} \bar{g}_{0, s}\right)+\frac{1}{2} \Delta t \partial_{t} \bar{g}_{0, s}\right) \vec{\Psi} d \Xi \\
& +\frac{\Delta t}{\tau}\left(\vec{W}_{i, \alpha}^{* n+1}-\vec{W}_{i, \alpha}^{n+1}\right)
\end{aligned}
$$

It can be observed that the numerical flux of conservative variables is consistent with the Navier-Stokes flux given by first order Chapman-Enskog expansion Eq. (9). Therefore in the continuum regime, the UGKWP method converges to Eq. (37), which is a second order gas-kinetic Navier-Stokes solver [39], i.e., the same as the direct macroscopic NS solver in smooth flow region.

3 As $\tau \rightarrow 0$, the total mass of simulation particle $e^{-\Delta t / \tau} \rho^{h} \Omega_{x} \rightarrow 0$, and therefore the number of simulation particles $N_{p} \rightarrow 0$ in continuum regime. As $\tau \rightarrow 0$, the total degree of freedom $N_{f}=N_{f}^{h}+N_{p} \rightarrow N_{f}^{h}$, and the UGKWP method is an asymptotic complexity diminishing scheme. 


\subsection{Asymptotic preserving property of UGKWP method for plasma transport}

Property 4.1 states that the UGKWP method for plasma transport preserves the two fluid model in the hydrodynamic regime. In this subsection, the behavior of the UGKWP method in the highly magnetized regime is discussed.

Proposition 4.2 In the highly magnetized regime as $r \rightarrow 0, \lambda_{D}=c^{-1}$, the linear system Eq. (35) is consistent to the magnetohydrodynamic equations.

Proof The Crank-Nicolson scheme for electromagnetic wave propagation gives

$$
\vec{E}^{n+1}-\vec{E}^{n}=\Delta t c^{2} \nabla \times \vec{B}+O\left(\Delta t^{2}, \Delta x^{2}\right) .
$$

The implicit discretization of the macroscopic interaction equations Eq. (35) gives

$$
\begin{aligned}
\vec{j}_{i}^{n+1}+\vec{j}_{e}^{n+1} & =\frac{\Delta t}{r} \lambda_{D}^{2} c^{2}\left(\vec{E}^{n+1}-\vec{E}^{n}\right) \\
& =r \nabla \times \vec{B}+O\left(\Delta t^{2}, \Delta x^{2}\right),
\end{aligned}
$$

and therefore the total momentum equation gives

$$
\begin{aligned}
\rho^{n+1} \vec{U}^{n+1}-\rho^{n+1} \vec{U}^{n} & =\frac{\Delta t}{r}\left[\left(\vec{j}_{i}^{n+1}+\vec{j}_{e}^{n+1}\right) \times \vec{B}^{n+1}\right] \\
& =\Delta t \nabla \times \vec{B} \times \vec{B}+O\left(\Delta t^{2}, \Delta x^{2}\right),
\end{aligned}
$$

which converges to a consistent MHD scheme.

\section{Numerical tests}

Five numerical tests are carried out in this section to verify the performance of the UGKWP method in various flow regimes, including three 1D and two 2D tests. Firstly, the shock structure of binary gas mixture is calculated to show the capability of the UGKWP method in capturing the highly non-equilibrium state inside the shock layer. The second test is the Landau damping and two steam instability, showing that the scheme can accurately capture the interaction between plasma and electromagnetic field. The Brio- Wu and Orszag-Tang tests verify the performance of the UGKWP method in different flow regimes. In the last, the scheme is applied to the magnetic reconnection problem to study how the electron-ion collision affects the reconnection rate. The code is sequentially operated on a single core of an i7-7660U CPU. The computational efficiency of the UGKWP method is lower than that of the UGKS in the one dimensional and rarefied regime flow calculations. For the simulations of two- and three-dimensional flows, especially close to the continuum regime, the UGKWP method is much more effective than the UGKS.

\subsection{Shock structure of binary gas mixture}

Normal shock structure is a standard test for verifying the scheme in capturing the nonequilibrium effect in rarefied regime. In this test, the Mach number is set as $M=1.5$, the mass ratio of gas mixture is $m_{B} / m_{A}=0.5$, diameter ratio $d_{B} / d_{A}=1$, and the component concentration of $\mathrm{B}$ is $\chi_{B}=0.1$. The hard sphere model is used and the reference mean free path is defined by

$$
\lambda_{\infty}=\frac{1}{\sqrt{2} \pi d_{A}^{2} n_{1}} .
$$


For each component, the upstream and downstream conditions are related through Rankine-Hugoniot condition. The cell size is chosen to be $\Delta x=0.5 \lambda_{\infty}$, and the CFL number is 0.95 . The mass of simulation particle is $m_{p, \alpha}=5 \times 10^{-3}$, which corresponds to around 200 simulation particles per cell. The normalized density, velocity and temperature are compared to the reference UGKS solution [40], as shown in Fig. 2. The UGKWP results well agree with the UGKS solution, which shows the capability of the UGKWP in capturing the non-equilibrium flow physics. The velocity space of the UGKS is set as $\left[-10 \sqrt{2 K_{B} T_{\alpha} / m_{\alpha}}, 10 \sqrt{2 K_{B} T_{\alpha} / m_{\alpha}}\right.$ for each species $\alpha$ and discretized into 100 velocity grids each. The computational time for the UGKS is 200 s to reach a steady state at the dimensionless physical time $t=250$. The computational time for the UGKWP is $5 \mathrm{mins}$, including 100 physical time to average the solution.

\subsection{Landau damping and two steam instability}

The Landau damping and two steam instability are two classical phenomena that have been well studied theoretically, and therefore we choose these two cases to test the accuracy of the UGKWP method in capturing the interaction between plasma and electromagnetic field. First we consider the Landau damping, which is about the VlasovPoisson system perturbed by a weak signal. The linear theory of Landau damping can be applied to predict the linear decay of electric energy with time [6]. The initial condition of linear Landau damping is

$$
f_{0}(x, u)=\frac{1}{\sqrt{2 \pi}}(1+\alpha \cos (k x)) \mathrm{e}^{-\frac{u^{2}}{2}}
$$

with $\alpha=0.01$. The length of the domain in the $\mathrm{x}$ direction is $L=2 \pi / k$. The background ion distribution function is fixed, uniformly chosen so that the total net charge density for the system is zero. When perturbation parameter $\alpha=0.01$ is small enough, the VlasovPoisson system can be approximated by linearization around the Maxwellian equilibrium. The analytical damping rate of electric field can be derived accordingly. Numerical cell number in physical space is $N_{x}=256$, and the particle number in each cell is $N_{p}=1000$. We test our scheme with different wave numbers and compare the numerical damping rates with theoretical values. For wave numbers $k=0.3$ and $k=0.4$, the evolution of
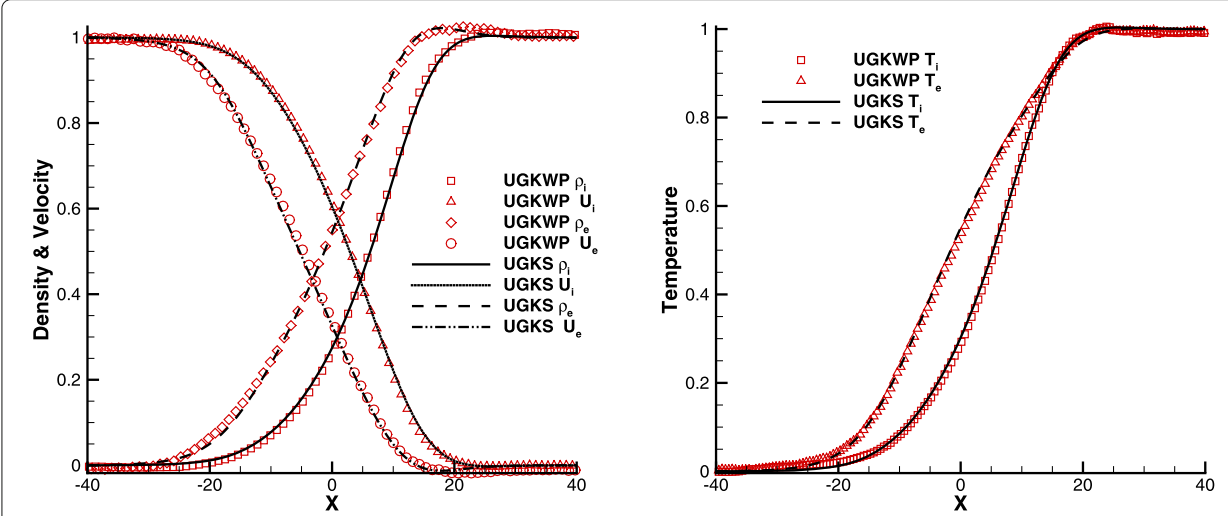

Fig. 2 Results of the shock wave in a binary gas mixture. Left figure shows the normalized density and velocity. Right figure shows the normalized temperature. Symbols show the UGKWP solution and lines show the reference $U$ GKS solution 

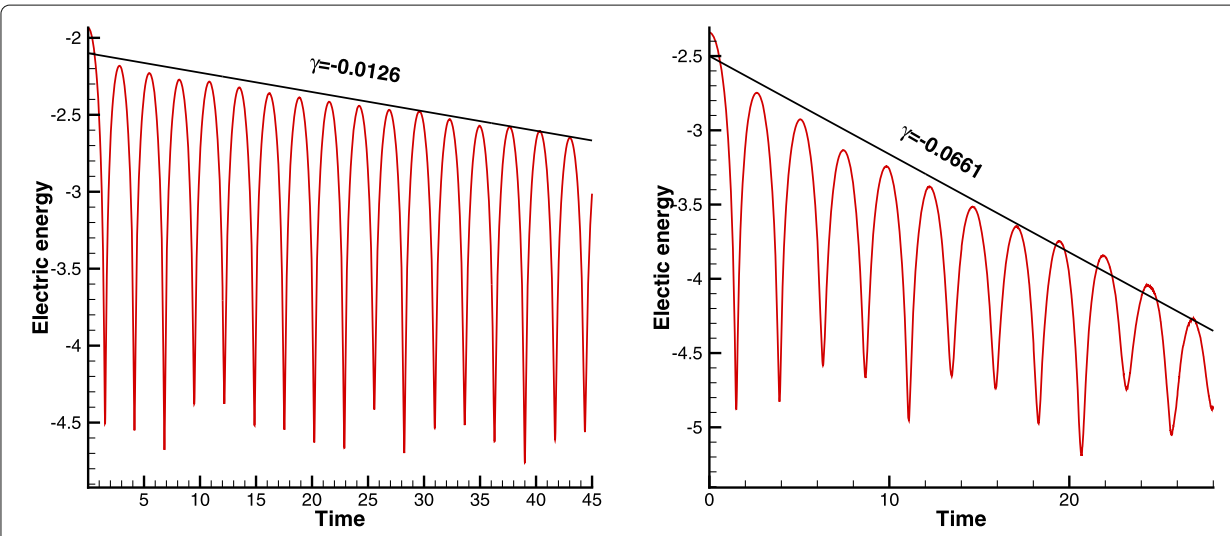

Fig. 3 Results of Landau damping. Red line shows the time evolution of the electric energy calculated by the UGKWP method, and the black lines give the theoretical prediction of the electric energy decay rate

the $L^{2}$ norm electric field is plotted in Fig. 3. It can be observed that the decay rates and oscillating frequencies $\omega=1.16,1.29$ agree well with theoretical data.

Once a larger perturbation with $\alpha=0.5$ and $k=0.5$ is applied, the linear theory breaks down, and the nonlinear phenomenon occurs. The evolution of the electric energy calculated by the UGKWP method is shown in Fig. 4a. The linear decay rate of electric energy is approximately equal to $\gamma_{1}=-0.287$, which agrees well with the values obtained by Heath et al. [41]. The growth rate predicted by the UGKWP method is approximately $\gamma_{2}=0.078$, which is between the values of 0.0815 computed by Rossmanith and Seal and 0.0746 by Heath et al. [42].

Next we consider the linear two stream instability problem with initial condition

$$
f(x, v, t=0)=\frac{2}{7 \sqrt{2 \pi}}\left(1+5 v^{2}\right)(1+\alpha((\cos (2 k x)+\cos (3 k x)) / 1.2+\cos (k x))) \mathrm{e}^{-\frac{v^{2}}{2}},
$$
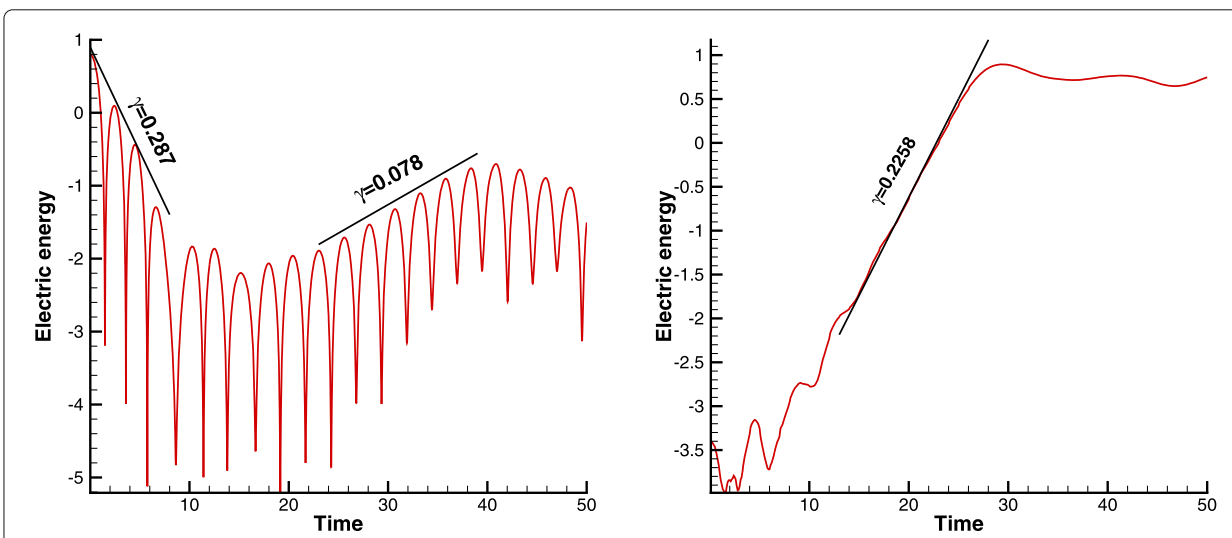

Fig. 4 Left figure shows the result of nonlinear Landau damping, Red line shows the time evolution of the electric energy calculated by the UGKWP method, and the black lines show the reference solutions. Right figure shows the result of two stream instability. Red line shows the time evolution of the electric energy calculated by the UGKWP method, and the black line gives the theoretical prediction of the electric energy increase rate 

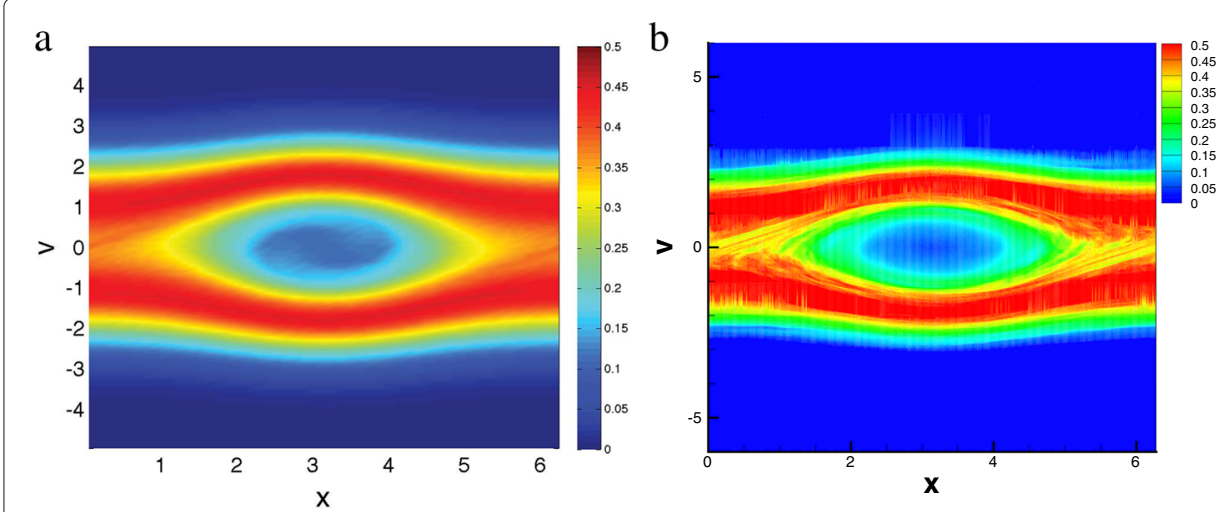

Fig. 5 Contour of the velocity distribution of the linear two stream instability at $t=70$. Left figure shows the WENO result and right figure shows the UGKWP solution

with $\alpha=0.001$ and $k=0.2$, and the nonlinear two stream instability problem with initial condition

$$
f(x, v, t=0)=\frac{1}{2 v_{t} \sqrt{2 \pi}}\left[\exp \left(-\frac{(u-U)^{2}}{2 v_{t}^{2}}\right)+\exp \left(-\frac{(u+U)^{2}}{2 v_{t}^{2}}\right)\right](1+\alpha \cos (k x)),
$$

with $\alpha=0.05, U=0.99, v_{t}=0.3$, and $k=2 / 13$. The length of the domain in the $\mathrm{x}$ direction is $L=\frac{2 \pi}{k}$. The background ion distribution function is fixed, uniformly to balance the charge density of electron. For the linear two stream instability a linear growth rate of electric field can be theoretically predicted [6]. The UGKWP method is applied to simulate this two stream instability problem with physical cell number $N_{x}=512$ and simulation particle number $N_{p}=1000$ per cell. The time evolution of electric energy is shown in Fig. 4b, and good agreement between the UGKWP solution and theoretical result can be observed. For the linear case, the distribution function contours in the phase space at $t=70$ are shown in Fig. 5 . And the contours of the nonlinear case at $t=70$ are shown in Fig. 6. The UGKWP results are compared with the WENO results [15]. Due to the statistical noise, deviation can be found in the distribution functions calculated by WENO and UGKWP. The overall resolution of the UGKWP is higher than the WENO results due to the Lagrangian nature in the particle method.
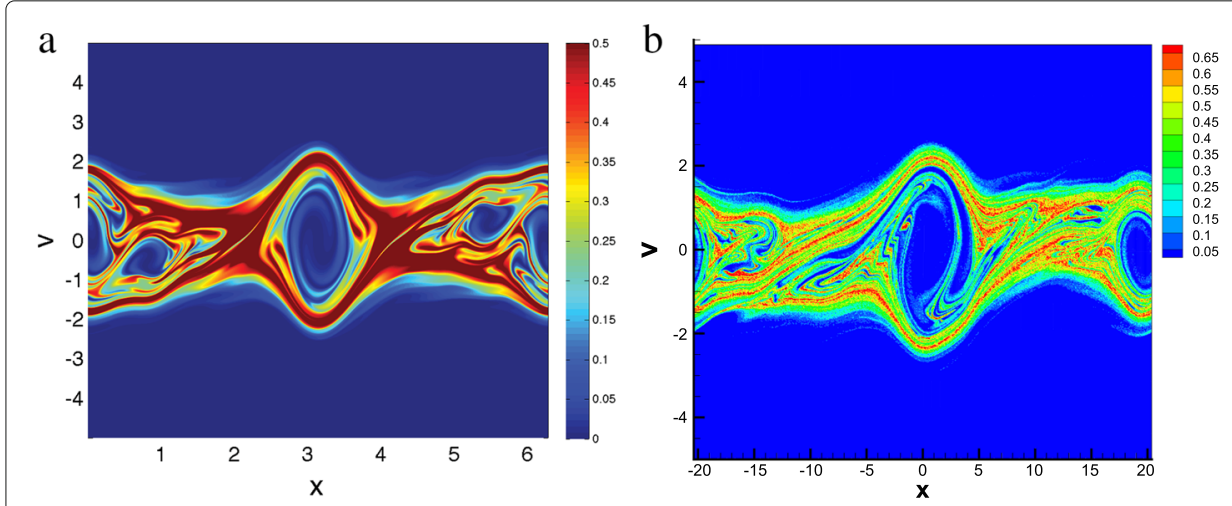

Fig. 6 Contour of the velocity distribution of the nonlinear two stream instability at $t=70$. Left figure shows the WENO result and right figure shows the UGKWP solution 


$$
\begin{gathered}
\rho_{i}=1.0, P_{i}=5.0 \times 10^{-5}, \vec{V}_{i}=0 ; \\
\rho_{e}=1.0 \frac{m_{e}}{m_{i}}, P_{e}=5.0 \times 10^{-5}, \vec{V}_{e}=0 ; \\
\vec{B}=(0.75,1.0,0) ; \vec{E}=0
\end{gathered}
$$$$
\rho_{i}=0.125, P_{i}=5.0 \times 10^{-6}, \vec{V}_{i}=0 ;
$$$$
\rho_{e}=0.125 \frac{m_{e}}{m_{i}}, P_{e}=5.0 \times 10^{-6}, \vec{V}_{e}=0
$$$$
\vec{B}=(0.75,-1.0,0) ; \vec{E}=0
$$

Fig. 7 Initial condition for the Brio-Wu shock tube problem

\subsection{Brio-Wu shock tube}

The Brio-Wu shock tube is originally designed for MHD solvers in continuum regime. Here we calculate the Brio-Wu problem in rarefied $(K n=1)$, transitional $\left(K n=10^{-2}\right)$, and continuum $\left(\mathrm{Kn}=10^{-4}\right)$ regimes. The same initial condition as the Brio-Wu one is given in Fig. 7. The ion to electron mass ratio is set to be 1836, and the ionic charge state is set to be unity. The normalized Debye length is $\lambda_{D}=0.001$, the normalized Larmor radius is $r=0.001$, and the normalized speed of light is 1000 . The grid points in physical space are $N_{x}=1000$. For the UGKS, the velocity space is discretized into 32 grids and for the UGKWP, the simulation particle mass is set as $m_{p}=10^{-5}$, which corresponds to about 100 particles per cell. The UGKWP solutions in rarefied, transitional, and continuum flow regimes are shown in Figs. 8, 9, and 10 and compared with the reference solutions of the UGKS. In the MHD regime, the UGKWP solution is shown in Fig. 11 and compared to

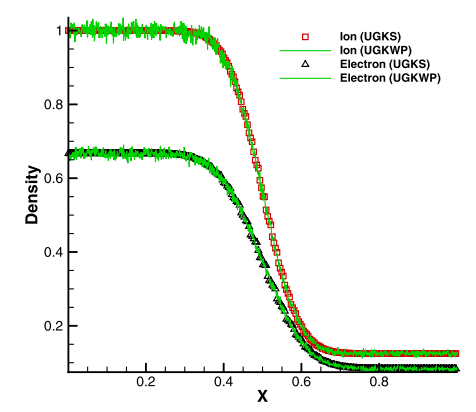

(a) Density distribution

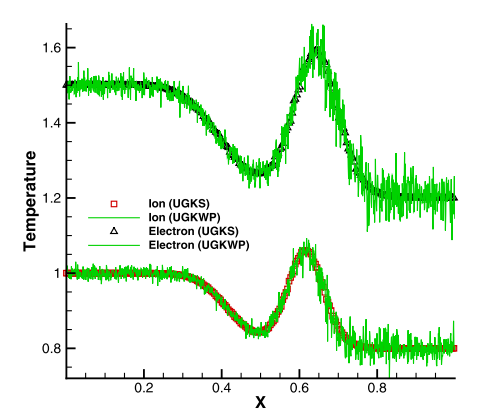

(c) Pressure distribution

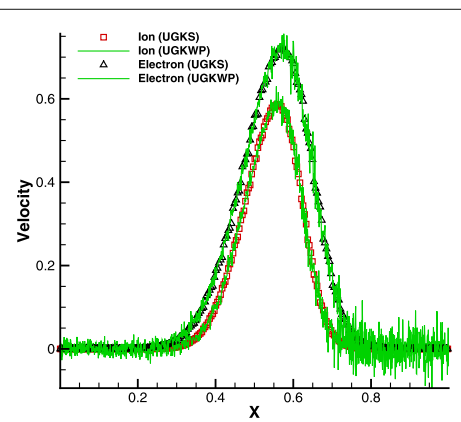

(b) X-velocity distribution

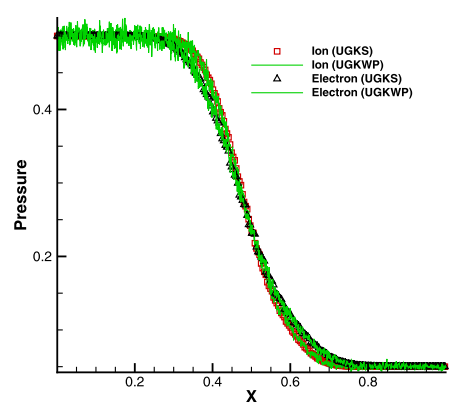

(d) Y-magnetic field distribution

Fig. 8 Multiscale Brio-Wu shock tube problem with $\mathrm{Kn}=1$ and $r=10^{-3}$. Lines show the UGKWP solutions and symbols show the reference UGKS solutions 


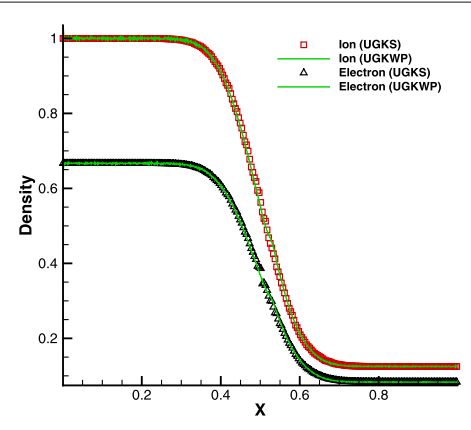

(a) Density distribution

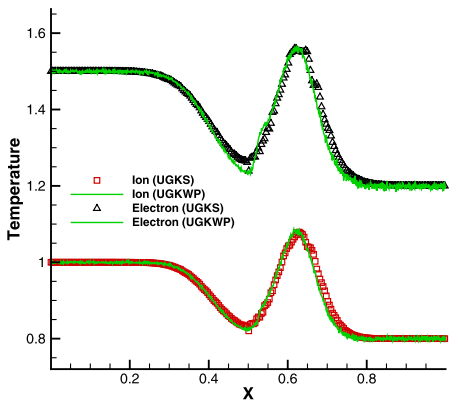

(c) Pressure distribution

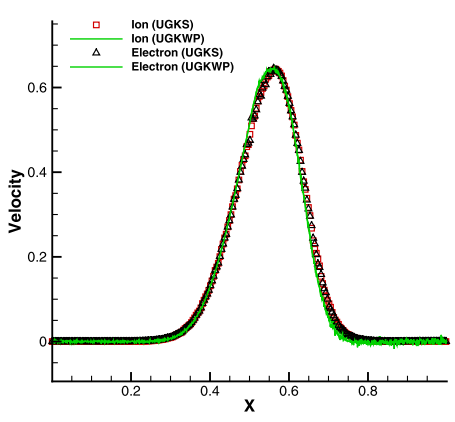

(b) X-velocity distribution

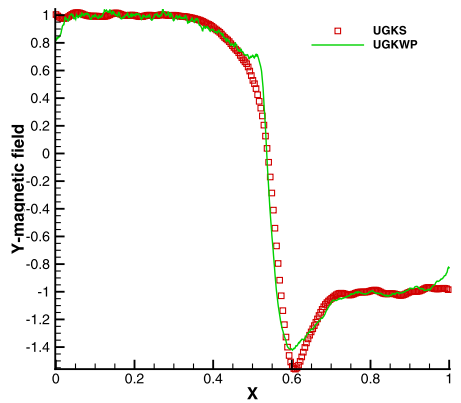

(d) Y-magnetic field distribution

Fig. 9 Multiscale Brio-Wu shock tube problem with $\mathrm{Kn}=10^{-2}$ and $r=10^{-3}$. Lines show the UGKWP solutions and symbols show the reference UGKS solutions

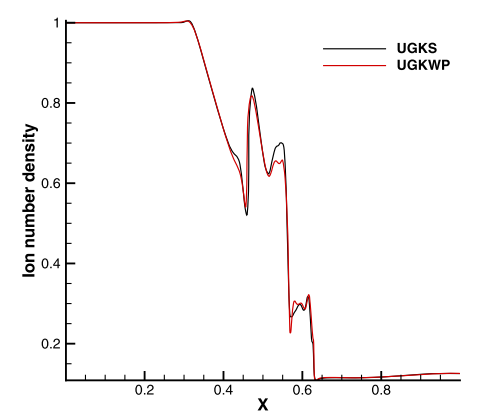

(a) Density distribution

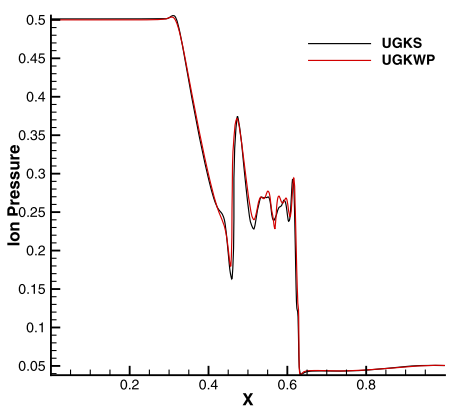

(c) Y-velocity distribution

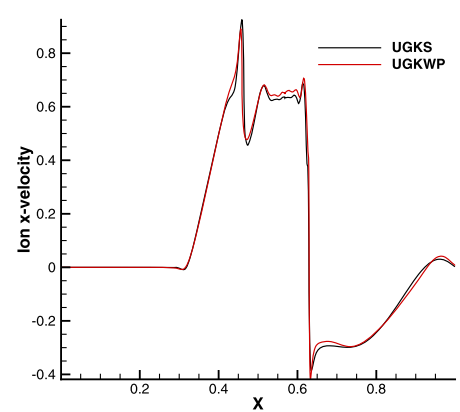

(b) X-velocity distribution

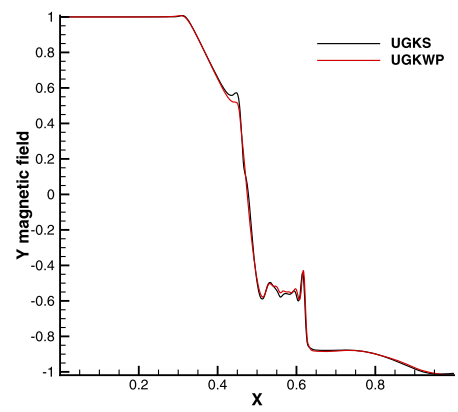

(d) Y-magnetic field distribution

Fig. 10 Multiscale Brio-Wu shock tube problem with $\mathrm{Kn}=10^{-4}$ and $r=10^{-3}$. Lines show the UGKWP solutions and symbols show the reference UGKS solutions 


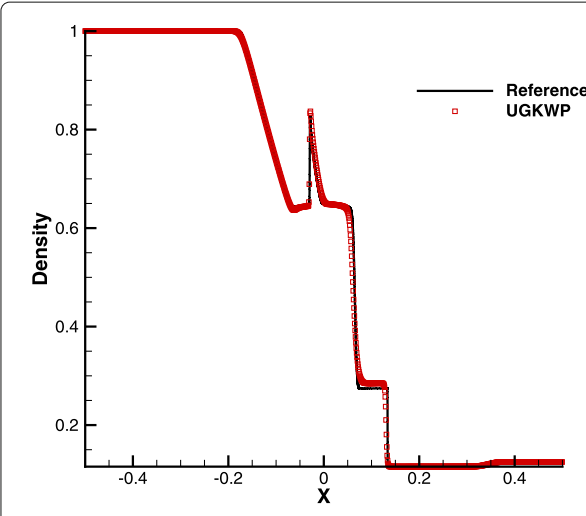

(a)Density distribution

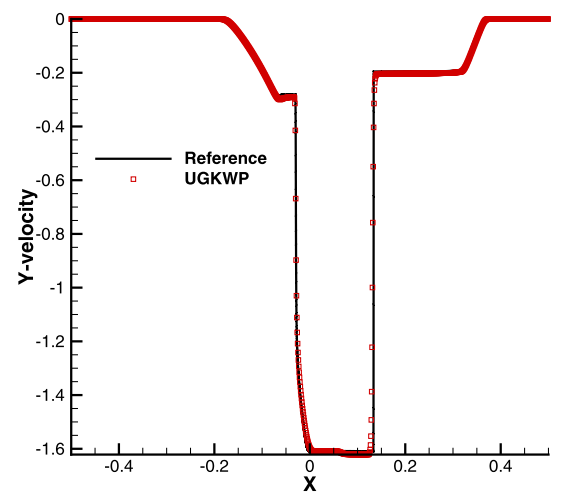

(c) Y-velocity distribution

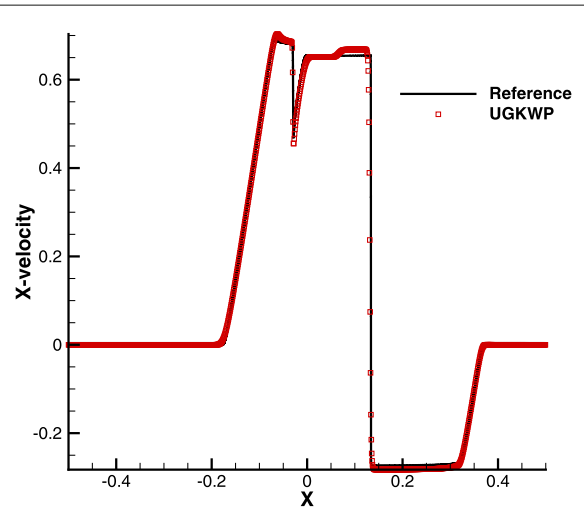

(b)X-velocity distribution

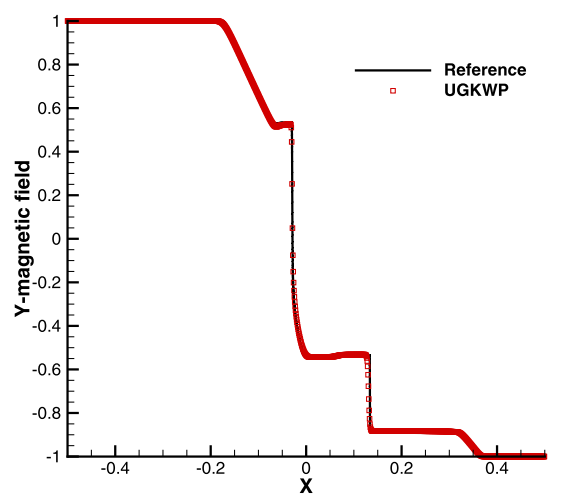

(d) Y-magnetic field distribution

Fig. 11 Multiscale Brio-Wu shock tube problem with $\mathrm{Kn}=10^{-4}$ and $r=0$. Lines show the UGKWP solutions and symbols show the reference MHD solutions

the reference ideal-MHD solution. In the rarefied regime, due to statistical noise, slight deviation can be observed between the UGKWP and the UGKS solutions, especially in the solutions of the magnetic field in the transitional regime, as shown in Fig. 9d. In the continuum regime, the UGKWP solution agrees well with the reference solution. It can be observed that the statistical noise significantly reduces as Knudsen number decreases due to the asymptotic complexity diminishing property of the UGKWP. In the rarefied regime with $\mathrm{Kn}=1$, the computational time for the UGKWP is 70mins with 50 times averaging, which is about 100 times slower than the UGKS. In the continuum regime with $\mathrm{Kn}=10^{-4}$, the computational time for the UGKWP is $5 \mathrm{~s}$ without averaging process, which is about 10 times faster than UGKS.

\subsection{Orszag-Tang vortex}

The Orszag-Tang Vortex problem was originally designed to study the MHD turbulence [43]. In this work, the problem is calculated in rarefied $(\mathrm{Kn}=1)$ and continuum $\left(\mathrm{Kn}=10^{-4}\right)$ regimes to verify the multiscale and asymptotic complexity diminishing property of the UGKWP. The initial condition for the current simulation is

$$
\begin{aligned}
& m_{i} / m_{e}=25, n_{i}=n_{e}=\gamma^{2}, P_{i}=P_{e}=\gamma, B_{y}=\sin (2 x), \\
& u_{i, x}=u_{e, x}=-\sin (y), u_{i, y}=u_{e, y}=\sin (x),
\end{aligned}
$$


where $\gamma=5 / 3$ and $r=0.001$. The computation domain is $[0,2 \pi] \times[0,2 \pi]$ with a uniform mesh of $200 \times 200$ cells. For the UGKWP, the mass of simulation particle is $m_{p}=10^{-5}$, which corresponds to around 100 particles per cell. For the UGKS, the velocity space is discretized into $32 \times 32$ velocity grids. The UGKWP result in rarefied regime is shown in Fig. 12 which is compared with the UGKS solution, and the UGKWP result in continuum regime is shown in Fig. 13. A better agreement and lower noise can be observed in the continuum regime due to the asymptotic preserving and the asymptotic complexity diminishing property of the UGKWP. In the MHD regime, the UGKWP solution with $\mathrm{Kn}=10^{-4}$ and $r=0$ is shown in Figs. 14, 15 and 16, where in Fig. 16b the UGKWP pressure distribution along $y=0.625 \pi$ is compared with the MHD solution [44]. The computational time of the UGKWP in the rarefied regime is 200mins with 10 times averaging, which is 5 times slower than the UGKS. In the continuum and MHD regime, the UGKWP takes around 2mins in computational time, which is close to the efficiency of hydrodynamic solver, and is significantly faster than the UGKS.

\subsection{Magnetic reconnection}

Magnetic reconnection is an important phenomenon that transfers magnetic energy into flow energy by topological change of magnetic field. In this test case, the UGKWP method is used to study the reconnection phenomenon in different flow regime, and study how the particle collision affects the collision rate and topology change of magnetic line. The
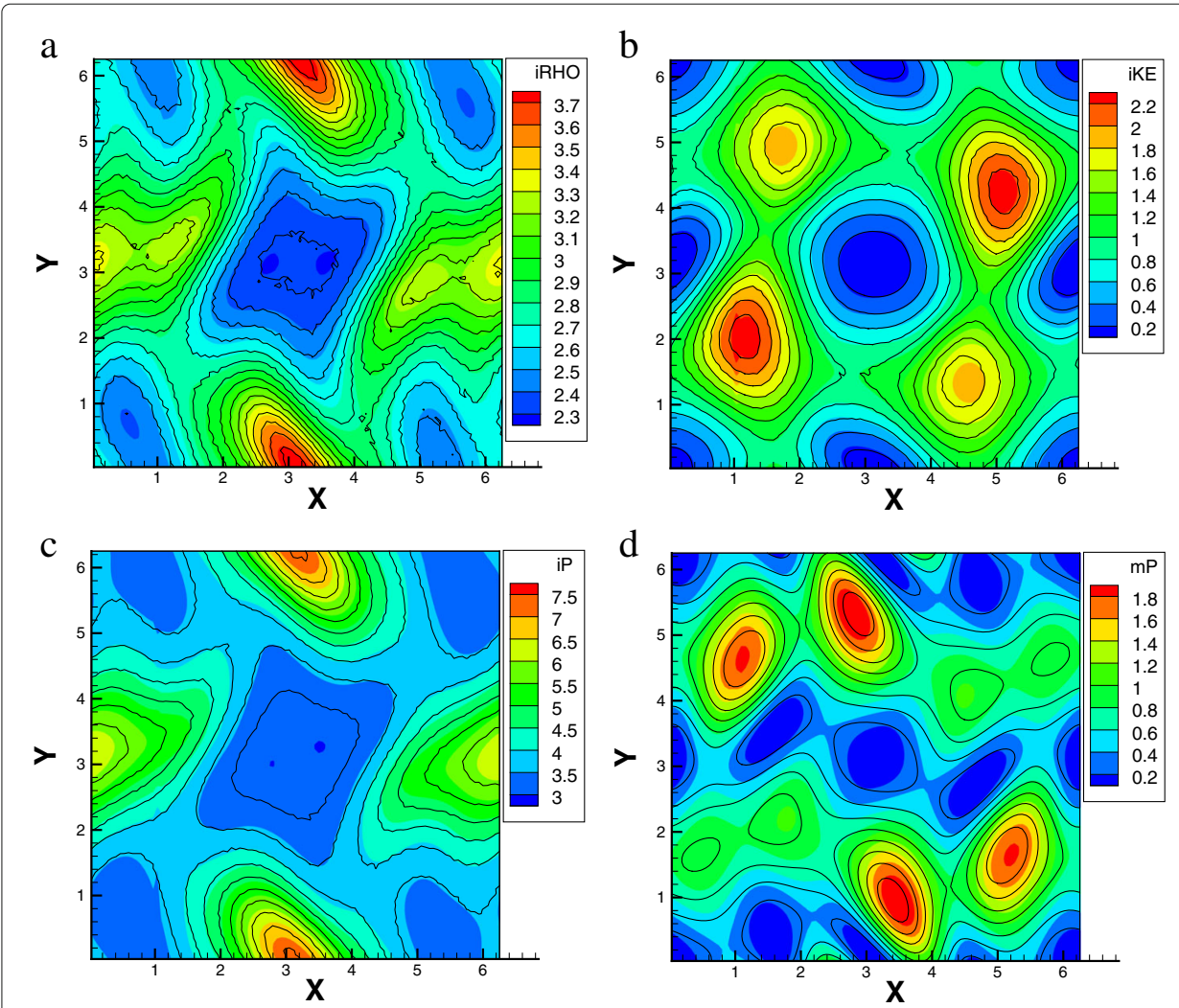

Fig. 12 The results of the multiscale Orszag-Tang vortex problem with $\mathrm{Kn}=1$ and $r=10^{-3}$ at $t=1$. Contour lines show the UGKWP solutions and contour floods show the UGKS solutions 

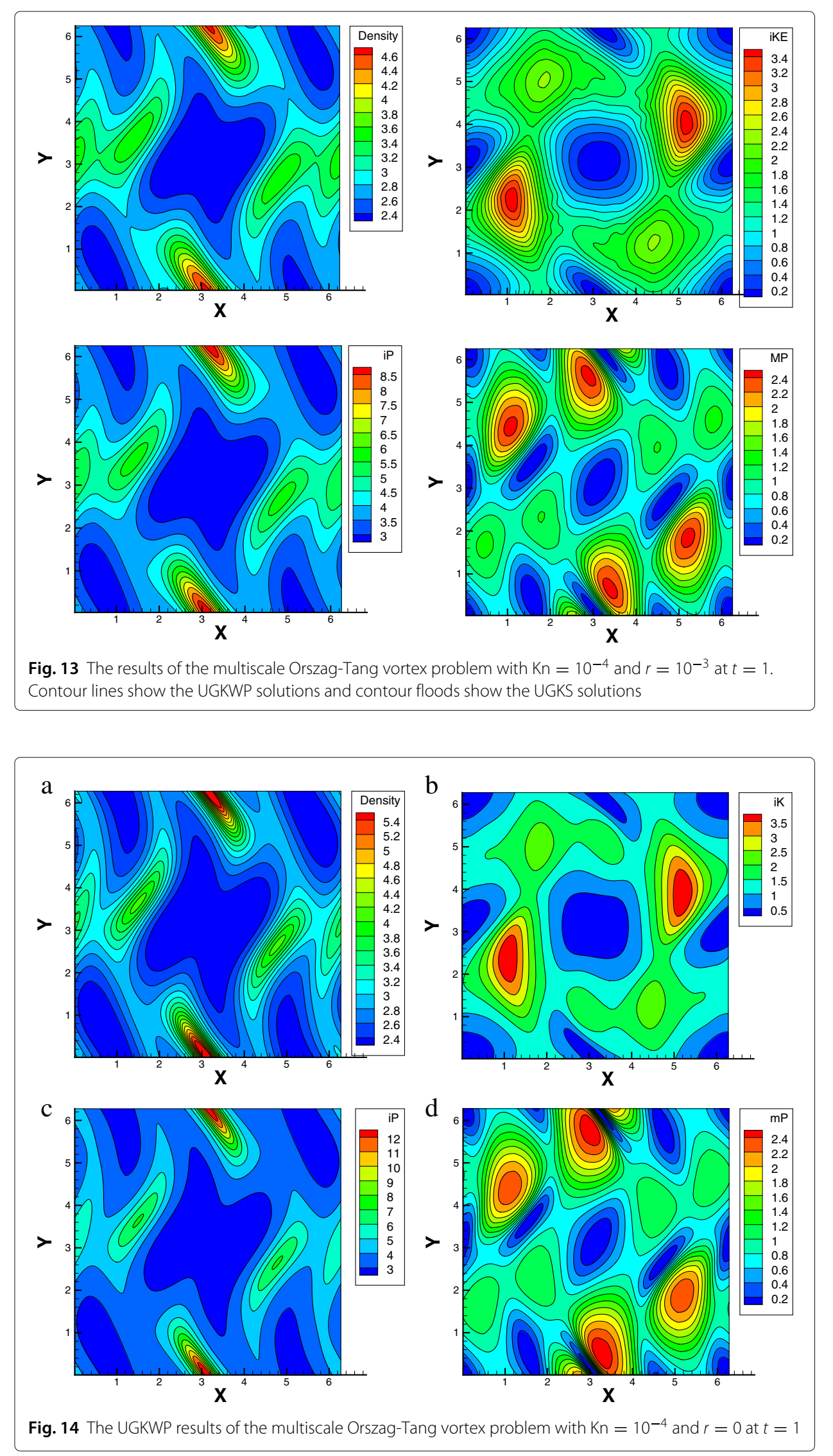

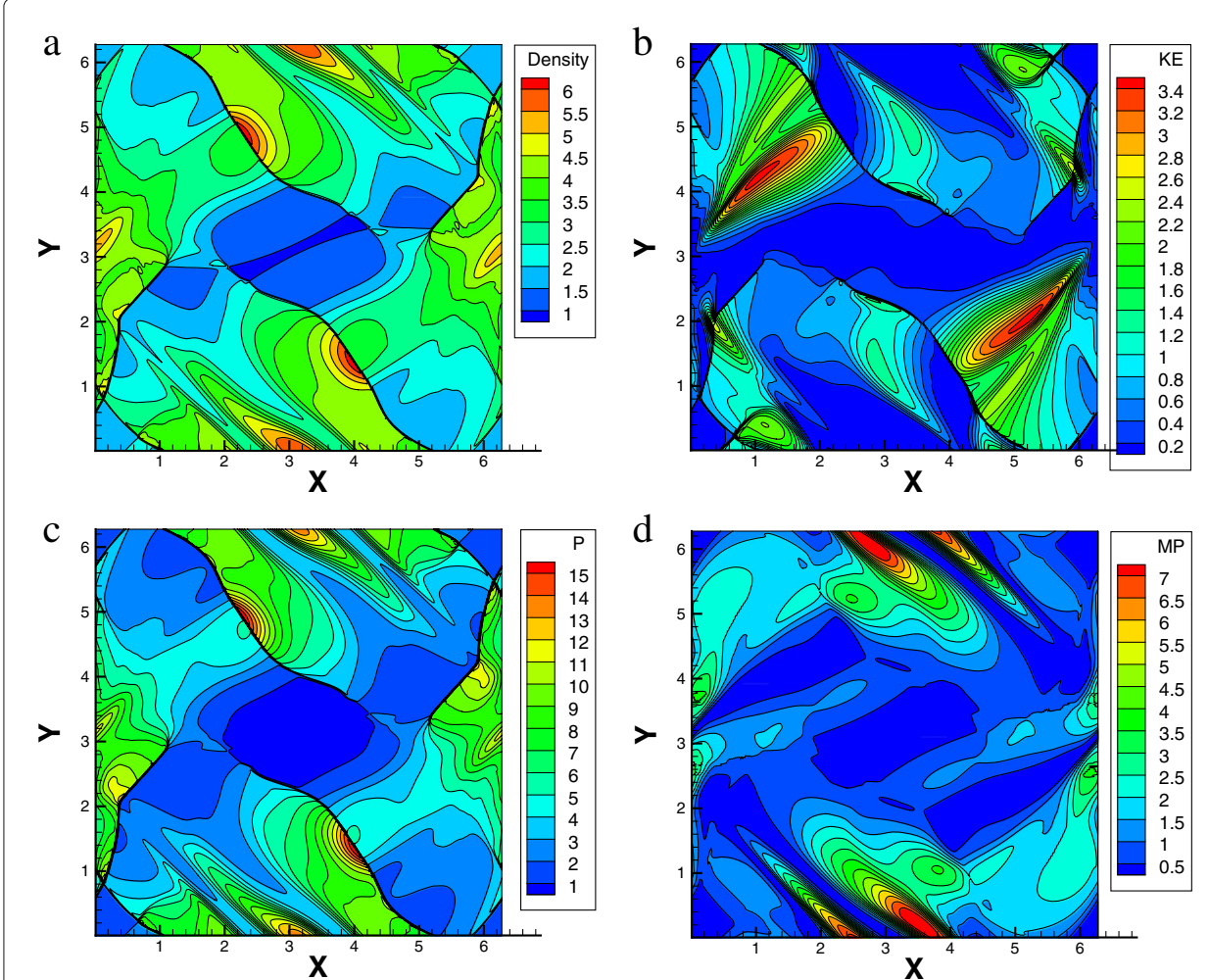

Fig. 15 The UGKWP results of the multiscale Orszag-Tang vortex problem with $\mathrm{Kn}=10^{-4}$ and $r=0$ at $t=2$

simulation uses the same initial condition as the GEM challenge problem [45]. The initial magnetic field is given by

$$
\vec{B}(y)=B_{0} \tanh (y / \lambda) \vec{e}_{x}
$$

and a corresponding current sheet is carried by the electrons
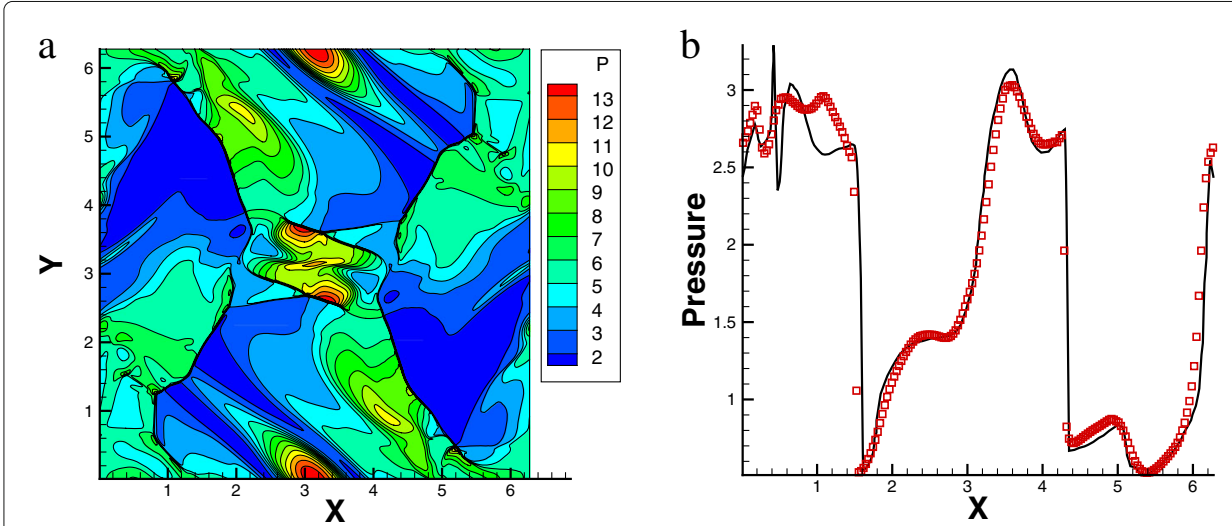

Fig. 16 The UGKWP results of the multiscale Orszag-Tang vortex problem with $\mathrm{Kn}=10^{-4}$ and $r=0$ at $t=3$. Sub-figure (a) shows the UGKWP pressure contour, and sub-figure (b) shows the comparison of the UGKWP and MHD pressure distribution along $y=0.625 \pi$ 


$$
\vec{J}_{e}=-\frac{B_{0}}{\lambda} \operatorname{sech}^{2}(y / \lambda) \vec{e}_{z}
$$

The initial number densities of electron and ion are

$$
n_{e}=n_{i}=1 / 5+\operatorname{sech}^{2}(y / \lambda)
$$

The electron and ion pressures are set to be

$$
P_{i}=5 P_{e}=\frac{5 B_{0}}{12} n(y)
$$

where $B_{0}=0.1, m_{i}=25 m_{e}$ and $\lambda=0.5$. The computational domain is $\left[-L_{x} / 2, L_{x} / 2\right] \times$ $\left[-L_{y} / 2, L_{y} / 2\right]$ with $L_{x}=8 \pi, L_{y}=4 \pi$, which is divided into $200 \times 100$ cells. Periodic boundaries are applied at $x= \pm L_{x} / 2$ and conducting wall boundaries at $y= \pm L_{y} / 2$. To initiate reconnection, the magnetic field is perturbed with $\delta \vec{B}=\vec{e}_{z} \times \nabla_{x} \psi$, where

$$
\psi(x, y)=0.1 B_{0} \cos \left(2 \pi x / L_{x}\right) \cos \left(\pi y / L_{y}\right) .
$$

Two Knudsen numbers are considered, $\mathrm{Kn}=10^{-3}$ in the transitional regime and $\mathrm{Kn}=10^{-4}$ in the continuum regime. The magnetic field topology as well as the distribution of flow variables in transitional regime is shown in Figs. 17-18 at $\omega_{p i} t=15$ and $\omega_{p i} t=30$, and the magnetic field topology in continuum regime as well as the magnetic reconnection rate is shown in Fig. 19. In the continuum regime, the topology of the magnetic field is symmetric, while in the transitional regime a magnetic island appears in the middle region at $\omega_{p} t=15$ and merges into the big right island at $\omega_{p} t=30$. Due to the magnetic island, two $\mathrm{x}$-shape reconnection points form and the reconnection rate in the transitional regime is significantly increased at $15<\omega_{p} t<30$. After $\omega_{p} t=30$ when the middle magnetic island merges with the right one, the reconnection rate slows down to the same reconnection intensity as in the continuum regime, which is shown in Fig. 19b.

\section{Conclusion}

In this work, we extend the unified gas-kinetic wave-particle method to the field of multispecies gas mixture and multiscale plasma transport. The construction of numerical scheme for multiscale transport is based on the direct modeling methodology [23], where the flow physics is modeled on the cell size and time step scales. In the unified framework, the evolution of microscopic velocity distribution function is coupled with the evolution of macroscopic quantities in a discretized space. The evolution solution of microscopic distribution function is modeled from the accumulating effect of particle transport and collision within a time step, from which numerical fluxes for both macroscopic flow variables and particle distribution function are obtained. The intrinsic governing equation underlying the unified scheme depends on the local cell's Knudsen number. A smooth transition from the kinetic particle transport to the continuum hydrodynamic flow evolution can be recovered seamlessly with the variation of the cell's Knudsen number. For the multispecies and plasma transport, the UGKWP has the properties of second order unified preserving as well as the asymptotic computational complexity diminishing. In plasma transport, the UGKWP method provides a smooth transition from PIC method in the kinetic scale to the MHD flow solvers in the continuum regime. All kinds of MHD equations, such as the two fluid models and ideal MHD, become subsets of the UGKWP modeling. Compared to the discrete velocity method (DVM), the UGKWP is much effi- 
a
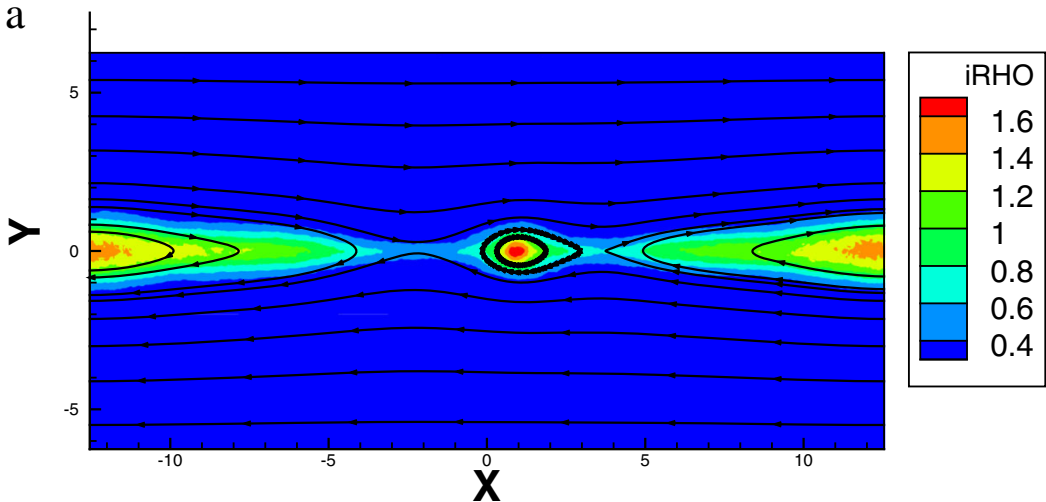

b

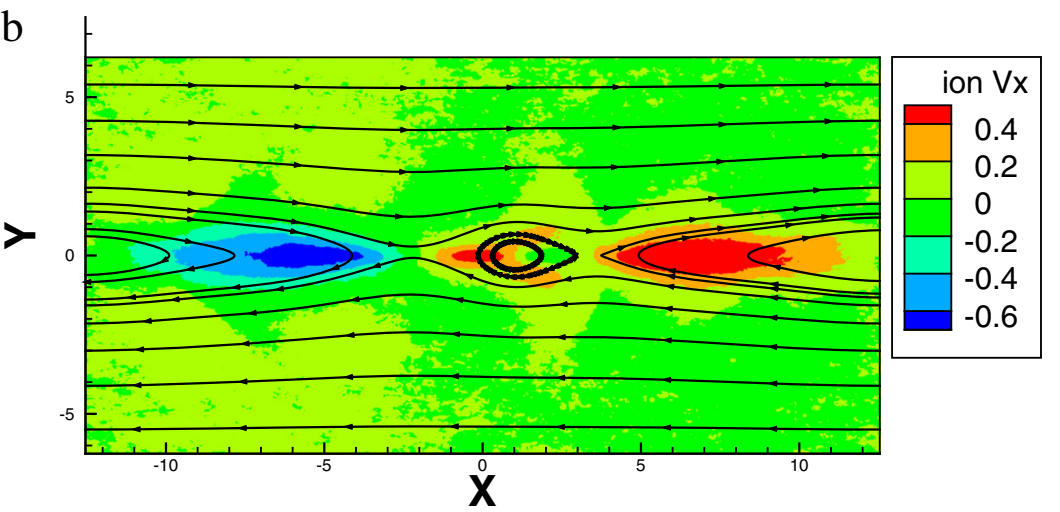

C
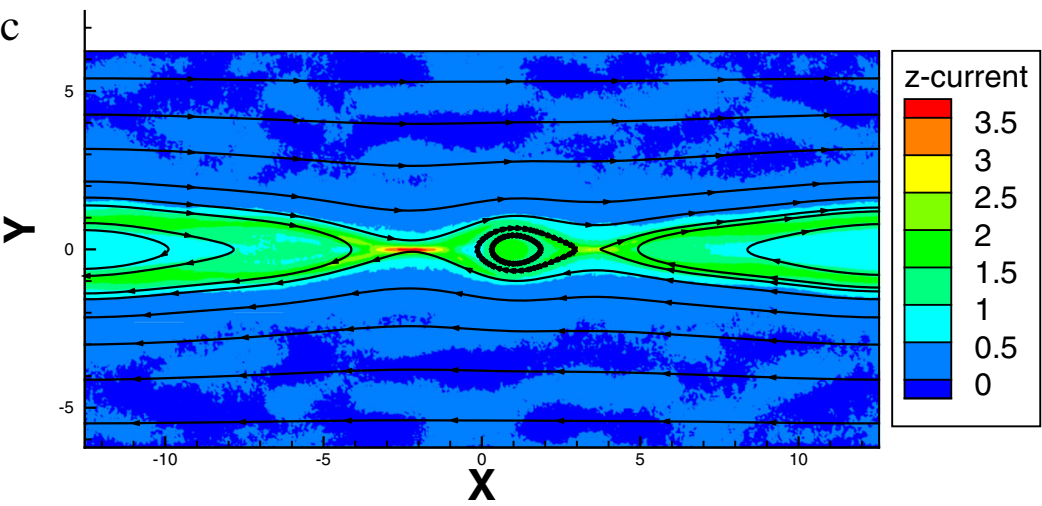

d

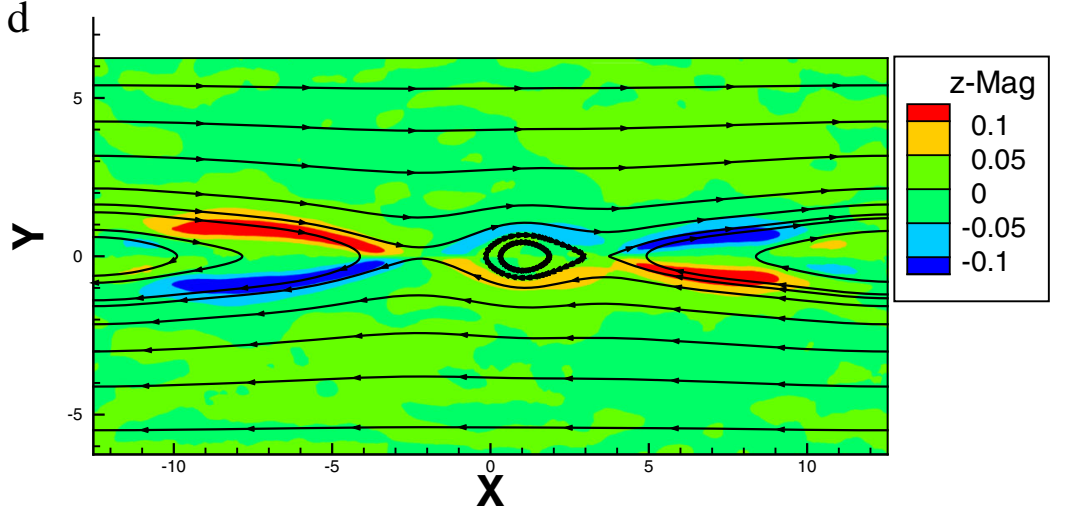

Fig. 17 The UGKWP results of magnetic reconnection with $K n=10^{-3}$ at $\omega_{p i} t=15$ 

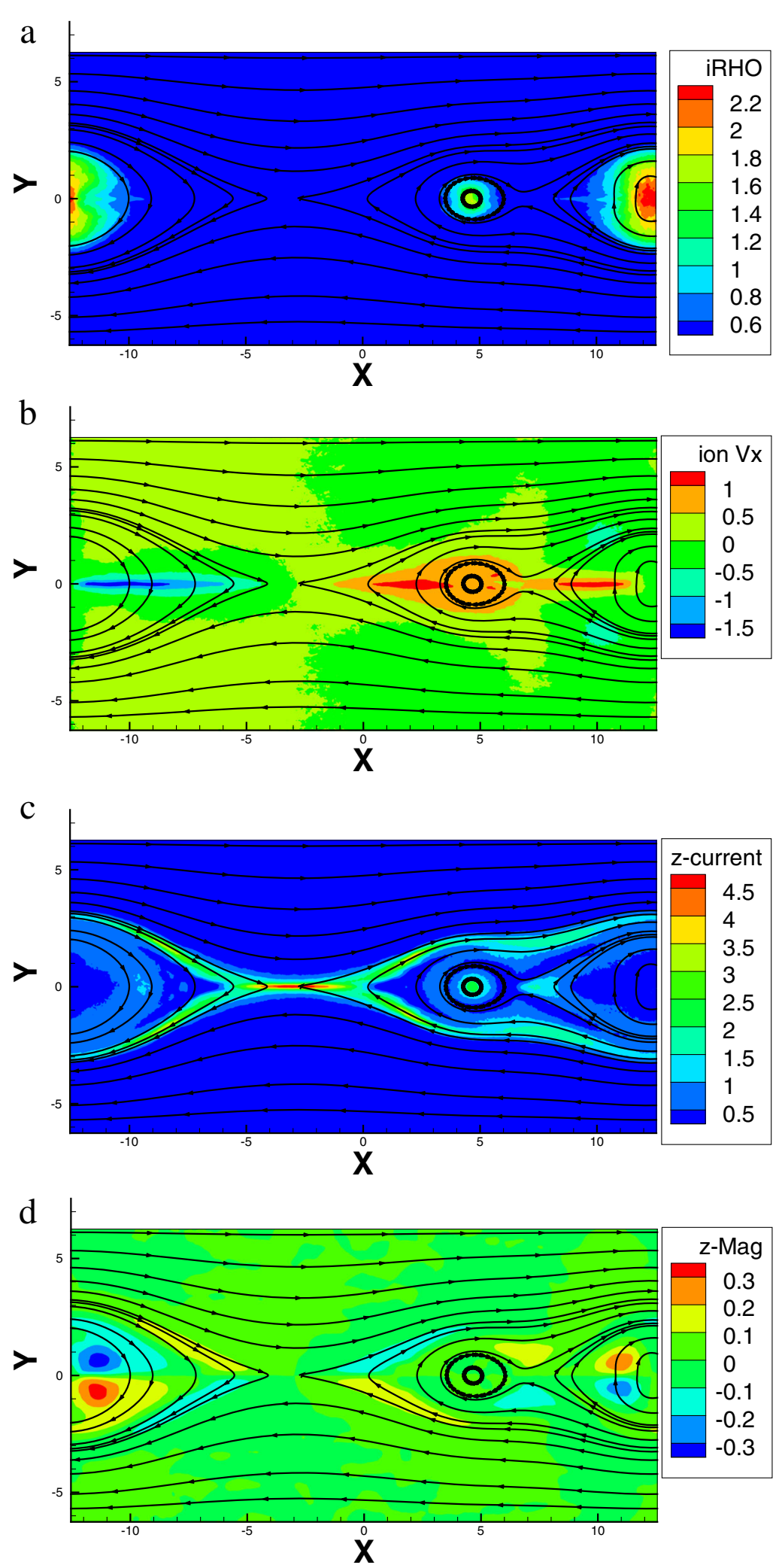

Fig. 18 The UGKWP results of magnetic reconnection with $K n=10^{-3}$ at $\omega_{p i} t=30$ 


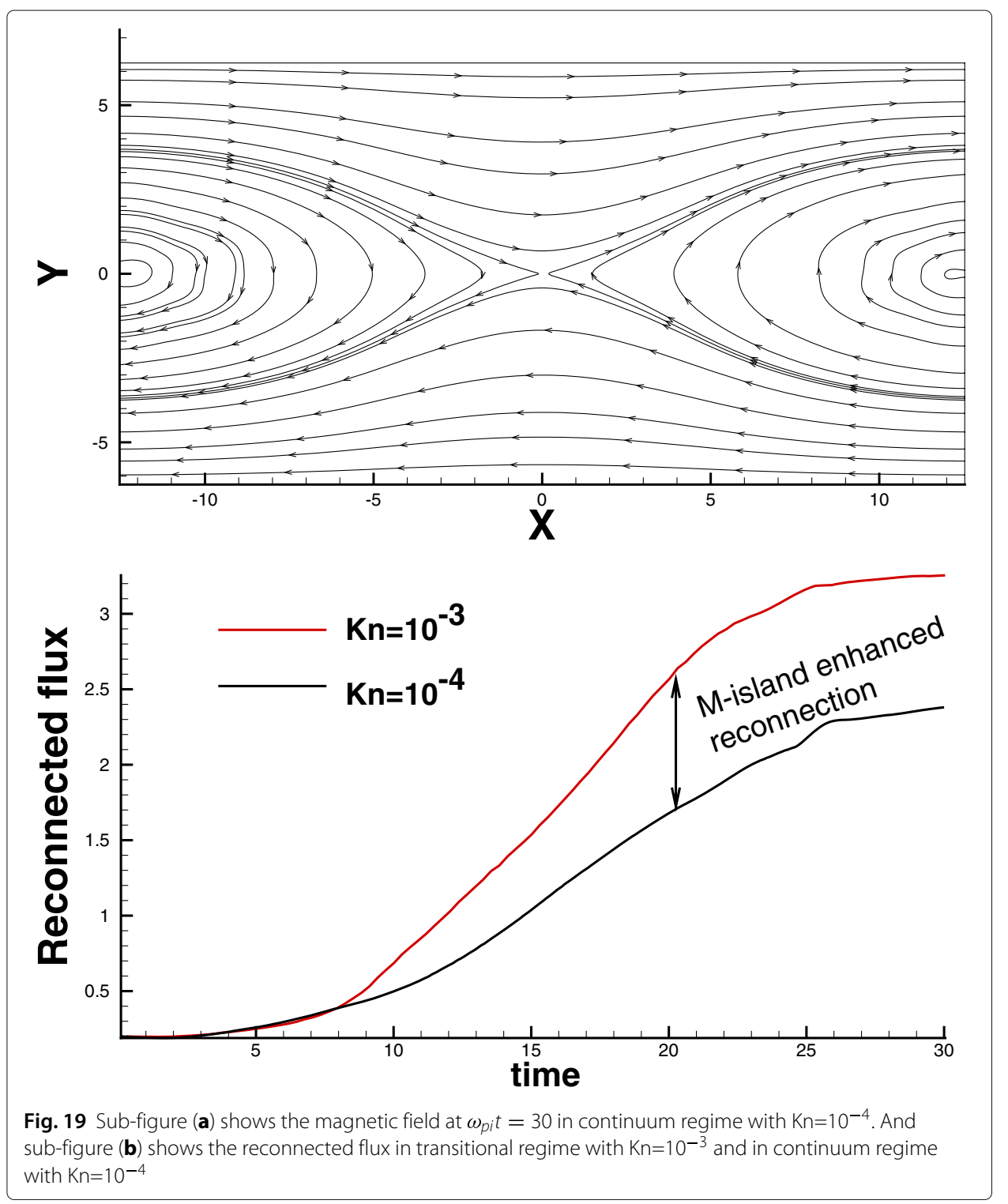

cient in the numerical simulation of highly non-equilibrium and high-dimensional flow problems, especially for the high speed flows. In conclusion, the UGKWP method has great potential to solve multiscale transport problems in rarefied flow [27, 28], radiative transfer $[29,46]$, and plasma physics.

Authors' contributions

Our group has been working on the topic for a long time. The research output is coming from our joint effort. All authors read and approved the final manuscript.

Funding

The current research is supported by National Numerical Windtunnel project and National Science Foundation of China $11772281,91852114$.

Availability of data and materials

All data and materials are available upon request.

\section{Competing interests}

The authors declare that they have no competing interests. 


\section{Author details}

${ }^{1}$ Department of Mathematics, Hong Kong University of Science and Technology, Hong Kong, China. ${ }^{2}$ Institute of Applied Physics and Computational Mathematics, Beijing 100088, China. ${ }^{3}$ Shenzhen Research Institute, Hong Kong University of Science and Technology, Shenzhen 518057, China.

Received: 12 September 2020 Accepted: 28 January 2021

Published online: 12 April 2021

\section{References}

1. Chapman S, Cowling TG, Burnett D (1990) The mathematical theory of non-uniform gases: an account of the kinetic theory of viscosity, thermal conduction and diffusion in gases. Cambridge university press, London

2. McCormack FJ (1973) Construction of linearized kinetic models for gaseous mixtures and molecular gases. Phys Fluids 16(12):2095-2105

3. Andries P, Aoki K, Perthame B (2002) A consistent BGK-type model for gas mixtures. J Stat Phys 106(5-6):993-1018

4. Brull S (2015) An ellipsoidal statistical model for gas mixtures. Commun Math Sci 13(1):1-13

5. Liu S, Liang Y (2016) Asymptotic-preserving Boltzmann model equations for binary gas mixture. Phys Rev E 93(2):023102

6. Chen FF (1984) Introduction to plasma physics and controlled fusion, Vol. 1. Springer, New York

7. Hakim A, Loverich J, Shumlak U (2006) A high resolution wave propagation scheme for ideal two-fluid plasma equations. J Comput Phys 219(1):418-442

8. Xu K (1999) Gas-kinetic theory-based flux splitting method for ideal magnetohydrodynamics. J Comput Phys 153(2):334-352

9. Liu C, Xu K (2020) A unified gas-kinetic scheme for micro flow simulation based on linearized kinetic equation. Adv Aerodyn 2:21. https://doi.org/10.1186/s42774-020-00045-8

10. Jin S, Shi Y (2010) A micro-macro decomposition-based asymptotic-preserving scheme for the multispecies Boltzmann equation. SIAM J Sci Comput 31(6):4580-4606

11. Zhang Y, Zhu L, Wang R, Guo Z (2018) Discrete unified gas kinetic scheme for all Knudsen number flows. III. Binary gas mixtures of Maxwell molecules. Phys Rev E 97(5):053306

12. Zhang Y, Zhu L, Wang P, Guo Z (2019) Discrete unified gas kinetic scheme for flows of binary gas mixture based on the McCormack model. Phys Fluids 31(1):017101

13. Brull S, Prigent C (2020) Local discrete velocity grids for multi-species rarefied flow simulations. Commun Comput Phys 28:1274-1304

14. Wu L, Zhang J, Reese JM, Zhang Y (2015) A fast spectral method for the Boltzmann equation for monatomic gas mixtures. J Comput Phys 298:602-621

15. Qiu J-M, Christlieb A (2010) A conservative high order semi-Lagrangian WENO method for the Vlasov equation. J Comput Phys 229(4):1130-1149

16. Liu C, Xu K (2017) A unified gas kinetic scheme for continuum and rarefied flows V: multiscale and multi-component plasma transport. Commun Comput Phys 22(5):1175-1223

17. Liu H, Shi F, Wan J, He X, Cao Y (2020) Discrete unified gas kinetic scheme for a reformulated BGK-Vlasov-Poisson system in all electrostatic plasma regimes. Comput Phys Commun 255:107400

18. Liu H, Quan L, Chen Q, Zhou S, Cao Y (2020) Discrete unified gas kinetic scheme for electrostatic plasma and its comparison with the particle-in-cell method. Phys Rev E 101(4):043307

19. Scanlon TJ, White C, Borg MK, et al (2015) Open source DSMC chemistry modelling for hypersonic flows. AIAA J 53(6):1670-1680. https://doi.org/10.2514/1.J053370

20. Tskhakaya D, Matyash K, Schneider R, Taccogna F (2007) The particle-in-cell method. Contrib Plasma Phys 47(8-9):563-594

21. Degond P, Deluzet F, Navoret L, Sun A-B, Vignal M-H (2010) Asymptotic-preserving particle-in-cell method for the Vlasov-poisson system near quasineutrality. J Comput Phys 229(16):5630-5652

22. Xu K, Huang J-C (2010) A unified gas-kinetic scheme for continuum and rarefied flows. J Comput Phys 229(20):7747-7764

23. Xu K (2015) Direct modeling for computational fluid dynamics: construction and application of unified gas-kinetic schemes. World Scientific, Singapore

24. Sun W, Jiang S, Xu K, Li S (2015) An asymptotic preserving unified gas kinetic scheme for frequency-dependent radiative transfer equations. J Comput Phys 302:222-238

25. Liu C, Wang Z, Xu K (2019) A unified gas-kinetic scheme for continuum and rarefied flows VI: Dilute disperse gas-particle multiphase system. J Comput Phys 386:264-295

26. Tan S, Sun W, Wei J, Ni G (2019) A parallel unified gas kinetic scheme for three-dimensional multi-group neutron transport. J Comput Phys 391:37-58

27. Liu C, Zhu Y, Xu K (2020) Unified gas-kinetic wave-particle methods I: Continuum and rarefied gas flow. J Comput Phys 401:108977

28. Zhu Y, Liu C, Zhong C, Xu K (2019) Unified gas-kinetic wave-particle methods. Il. multiscale simulation on unstructured mesh. Phys Fluids 31(6):067105

29. Li W, Liu C, Zhu Y, Zhang J, Xu K (2020) Unified gas-kinetic wave-particle methods III: Multiscale photon transport. J Comput Phys 408:109280

30. Sirovich L (1962) Kinetic modeling of gas mixtures. Phys Fluids 5(8):908-918

31. Garzó V, Santos A, Brey J (1989) A kinetic model for a multicomponent gas. Phys Fluids A: Fluid Dyn 1(2):380-383

32. Asinari P, Luo L-S (2008) A consistent lattice Boltzmann equation with baroclinic coupling for mixtures. J Comput Phys 227(8):3878-3895

33. Groppi M, Monica S, Spiga G (2011) A kinetic ellipsoidal BGK model for a binary gas mixture. Europhys Lett 96(6):64002

34. Gross EP, Krook M (1956) Model for collision processes in gases: Small-amplitude oscillations of charged two-component systems. Phys Rev 102(3):593 
35. Morse T (1963) Energy and momentum exchange between nonequipartition gases. Phys Fluids 6(10):1420-1427

36. Yang Y, Chen R, Yung EK (2006) The unconditionally stable Crank Nicolson FDTD method for three-dimensional Maxwell's equations. Microw Opt Technol Lett 48(8):1619-1622

37. Guo Z, Li J, Xu K (2020) On unified preserving properties of kinetic schemes. arXiv preprint arXiv:1909.04923. https:// arxiv.xilesou.top/abs/1909.04923

38. Crestetto A, Crouseilles N, Dimarco G, Lemou M (2019) Asymptotically complexity diminishing schemes (ACDS) for kinetic equations in the diffusive scaling. J Comput Phys 394:243-262

39. Xu K (2001) A gas-kinetic BGK scheme for the Navier-Stokes equations and its connection with artificial dissipation and Godunov method. J Comput Phys 171(1):289-335

40. Wang R (2015) Unified gas-kinetic scheme for the study of non-equilibrium flows. Dissertation, Hong Kong University of Science and Technology. https://pdfs.semanticscholar.org/1940/3e7da67f921f80deb785dab324164a5ac5a7.pdf

41. Heath R, Gamba I, Morrison P, Michler C (2012) A discontinuous Galerkin method for the Vlasov-Poisson system. J Comput Phys 231:1140-1174

42. Rossmanith JA, Seal DC (2011) A positivity-preserving high-order semi-Lagrangian discontinuous Galerkin scheme for the Vlasov-Poisson equations. J Comput Phys 230:6203-6232

43. Orszag SA, Tang C-M (1979) Small-scale structure of two-dimensional magnetohydrodynamic turbulence. J Fluid Mech 90(1):129-143

44. Tang H-Z, Xu K (2000) A high-order gas-kinetic method for multidimensional ideal magnetohydrodynamics. J Comput Phys 165:69-88

45. Birn J, Drake J, Shay M, Rogers B, Denton R, Hesse M, Kuznetsova M, Ma Z, Bhattacharjee A, Otto A, et al (2001) Geospace environmental modeling (GEM) magnetic reconnection challenge. J Geophys Res: Space Phys 106(A3):3715-3719

46. Shi Y, Song P, Sun W (2020) An asymptotic preserving unified gas kinetic particle method for radiative transfer equations. J Comput Phys 420:109687

\section{Publisher's Note}

Springer Nature remains neutral with regard to jurisdictional claims in published maps and institutional affiliations.

Ready to submit your research? Choose BMC and benefit from:

- fast, convenient online submission

- thorough peer review by experienced researchers in your field

- rapid publication on acceptance

- support for research data, including large and complex data types

- gold Open Access which fosters wider collaboration and increased citations

- maximum visibility for your research: over $100 \mathrm{M}$ website views per year

At BMC, research is always in progress.

Learn more biomedcentral.com/submissions 